\title{
Total Synthesis and Stereochemical Assignment of the Spiroisoxazoline Natural Product (+)-calafianin
}

\author{
Sujata Bardhan, Daniel C. Schmitt, and John A. Porco, Jr.* \\ Department of Chemistry and Center for Chemical Methodology and Library \\ development, (CMLD-BU), Boston University, Boston, Massachusetts 02215
}

\section{Supporting Information}

General Information: ${ }^{1} \mathrm{H}$ NMR spectra were recorded on a $400 \mathrm{MHz}$ spectrometer at ambient temperature with $\mathrm{CDCl}_{3}$ as the solvent unless otherwise stated. ${ }^{13} \mathrm{C}$ NMR spectra were recorded on a $75.0 \mathrm{MHz}$ spectrometer at ambient temperature. Chemical shifts are reported in parts per million relative to chloroform $\left({ }^{1} \mathrm{H}, \delta 7.24 ;{ }^{13} \mathrm{C}, \delta 77.23\right)$, acetone $\left({ }^{1} \mathrm{H}, \delta 2.09 ;{ }^{13} \mathrm{C}, \delta\right.$ 205. 87, 30.92), dimethyl sulfoxide $\left({ }^{1} \mathrm{H}, \delta 2.52 ;{ }^{13} \mathrm{C}, \delta 40.45\right)$, (Data for ${ }^{1} \mathrm{H}$ NMR are reported as follows: chemical shift, multiplicity $(\mathrm{s}=$ singlet, $\mathrm{d}=$ doublet, $\mathrm{t}=$ triplet, $\mathrm{q}=$ quartet, $\mathrm{m}=$ multiplet, br $=$ broad), integration, and coupling constants. All ${ }^{13} \mathrm{C}$ NMR spectra were recorded with complete proton decoupling. Infrared spectra were recorded on a Nicolet Nexus 670 FT-IR spectrophotometer for liquids and Avatar Smart MIRacle for solid compounds. Low and high-resolution mass spectra were obtained in the Boston University Mass Spectrometry Laboratory using Finnegan MAT-90 and Waters Q-TOF spectrometers. Optical rotations were recorded on an AUTOPOL III digital polarimeter at $589 \mathrm{~nm}$, and are recorded as $[\alpha]_{\mathrm{D}}^{22}$ (concentration in grams/100 mL solvent). Circular Dichroism experiments were run on a CD Spectrometer, Model 62DS from Aviv Lakewood, NJ. UV absorptions were recorded using a CARY 100 Bio UV Visible Spectrophotometer from Varian. Chiral HPLC analysis was performed on an Agilent 1100 series HPLC (CHIRALCEL OD, Column No. OD00CEAI015). Analytical thin layer chromatography was performed using $0.25 \mathrm{~mm}$ silica gel $60-\mathrm{F}$ plates. Flash chromatography was performed using 200-400 mesh silica gel (Sorbent Technologies.). Preparative glass plates (TLC) were supplied by Silicycle. Yields refer to chromatographically and spectroscopically pure materials, unless otherwise stated. Methylene chloride, toluene, hexane, and tetrahydrouran were distilled from columns. Methanol, acetonitrile $\mathrm{Zr}\left(\mathrm{O}^{\mathrm{t}} \mathrm{Bu}\right)_{4}$ was purchased from Strem Chemicals. $\mathrm{MgBr}_{2} \cdot \mathrm{Et}_{2} \mathrm{O}$ was freshly prepared following literature procedure. ${ }^{\mathrm{s}_{1}}$ Trityl hydroperoxide ${ }^{\mathrm{S}_{2}}$ was prepared from trityl alcohol according to a literature procedure. All other reagents and solvents were used as supplied by Sigma-Aldrich, Fluka, and Strem Chemicals. All reactions were

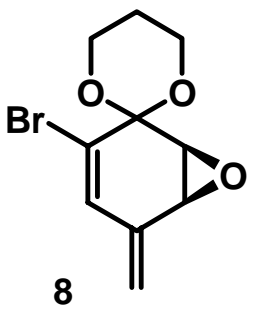
carried out in oven-dried glassware under an argon atmosphere unless otherwise noted.

Vinyl Epoxide (8): Methyl-triphenyl-phosphoniumbromide (7.13 g, $19.96 \mathrm{mmol}$ ) was placed in a round bottom flask and distilled THF (100 $\mathrm{mL}, 0.2 \mathrm{M})$ and $\mathrm{KO}^{\mathrm{t}} \mathrm{Bu}(2.01 \mathrm{~g}, 17.96 \mathrm{mmol})$ were added at rt. The

${ }^{\mathrm{S} 1}$ Organic Syntheses, Coll. Vol. 6, p.845 (1988); Vol. 59, p.85 (1979).

S2 D. E. Bissing, C. A. Matuszac, W. E. McEwen, J. Am. Chem. Soc. 1964, 86, 3824. 
reaction mixture was stirred for $3 \mathrm{~h}$. Epoxy ketone $\mathbf{1 0}^{\mathrm{S}_{3}}$ (1.04 g, $\left.3.99 \mathrm{mmol}\right)$ was dissolved in distilled THF $(30 \mathrm{~mL}, 0.13 \mathrm{M})$ and cooled to $-40{ }^{\circ} \mathrm{C}$. The yellow ylide solution was slowly added by cannula to the cooled reaction mixture containing $\mathbf{1 0}$ until a yellow color persisted. The reaction was immediately quenched with satd. $\mathrm{NH}_{4} \mathrm{Cl}$ and extracted with diethyl ether, concentrated, and subjected to silica gel chromatography $(30 \%$ ether/pet. Ether) to afford vinyl epoxide 8 as a white solid (0.98 g, 95\%). M.p. $66-67{ }^{\circ} \mathrm{C} .{ }^{1} \mathrm{H}$ NMR $\left(400 \mathrm{MHz}, \mathrm{CDCl}_{3}\right) \delta 6.53(\mathrm{~d}, 1 \mathrm{H}, J=2 \mathrm{~Hz}), 5.49(\mathrm{~s}, 1 \mathrm{H}), 5.379(\mathrm{~s}, 1 \mathrm{H}), 4.33-4.27(\mathrm{~m}, 2 \mathrm{H})$, $4.18(\mathrm{~d}, 1 \mathrm{H}, J=4 \mathrm{~Hz}), 4.15-4.10(\mathrm{~m}, 2 \mathrm{H}), 3.73(\mathrm{dd}, 1 \mathrm{H}, J=1.6 \mathrm{~Hz}, J=4 \mathrm{~Hz}), 2.31-2.19$ $(\mathrm{m}, 1 \mathrm{H}), 1.59-1.54,1.30-1.28(\mathrm{~m}, 1 \mathrm{H}) ;{ }^{13} \mathrm{C} \mathrm{NMR}\left(100 \mathrm{MHz}, \mathrm{CDCl}_{3}\right) \delta 136.6,131.0$, 125.7, 122.2, 93.0, 62.0, 60.9, 54.3, 51.4, 24.5; IR (neat) vmax 2971, 2876, 1684, 1643, 1404, 1381, 1283, 1204, $1041 \mathrm{~cm}^{-1}$; CIHRMS $[\mathrm{M}+\mathrm{H}]^{+}$calculated for $\mathrm{C}_{10} \mathrm{H}_{11} \mathrm{BrO}_{3}$ : 257.9892, found: $257.9877 .[\alpha]_{\mathrm{D}}^{22}=+293\left(\mathrm{c}=0.160, \mathrm{CHCl}_{3}\right)$.

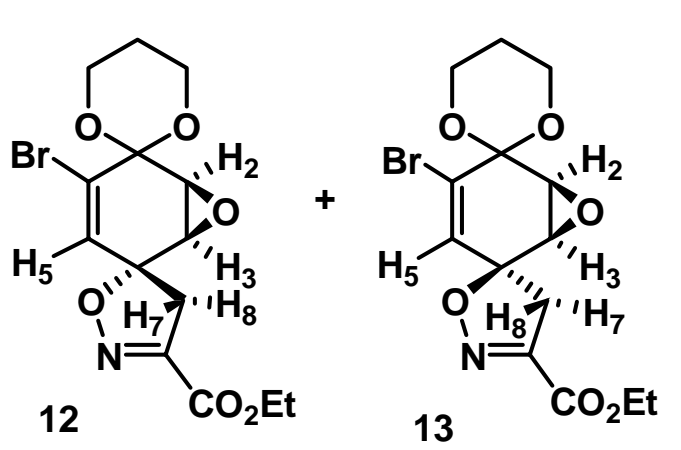

Anti-spiroisoxazoline ester (12): To vinyl epoxide 8 (300 $\mathrm{mg}, 1.16 \mathrm{mmol})$ in anhydrous toluene $(20 \mathrm{~mL}, 0.06 \mathrm{M})$ was added $\mathrm{Zr}(\mathrm{OtBu})_{4}$ (0.44 $\mathrm{mL}, 1.16 \mathrm{mmol})$ followed by ethyl chlorooximido acetate 11 (350 $\mathrm{mg}, 2.32 \mathrm{mmol}$ ) at room temperature. The mixture was stirred at $\mathrm{rt}$ for $24 \mathrm{~h}$ before being quenched with water. The mixture was extracted with EtOAc, the organic extracts were combined, washed with brine, dried over $\mathrm{MgSO}_{4}$, filtered, and concentrated in vacuo. Purification on silica gel (30\% EtOAc in hexane) provided $\mathbf{1 2}$ as a white solid (260 $\mathrm{mg}, 60 \%$ ) and 13 as a yellow solid (142 $\mathrm{mg}$, 33\%) (Combined yield: 93\%. 12:13=1.8:1.0).

(12): M.p. 80-82 ${ }^{0} \mathrm{C} .{ }^{1} \mathrm{H}$ NMR $\left(300 \mathrm{MHz}, \mathrm{CDCl}_{3}\right) \delta 6.06(\mathrm{~d}, 1 \mathrm{H}, J=2.1 \mathrm{~Hz}), 4.37$ (q, $2 \mathrm{H}, J=$ $7.2 \mathrm{~Hz}), 4.30-4.11(\mathrm{~m}, 4 \mathrm{H}), 4.10(\mathrm{~d}, 1 \mathrm{H}, J=4.2 \mathrm{~Hz}), 3.56(\mathrm{~d}, 1 \mathrm{H}, J=18.6 \mathrm{~Hz}), 3.47$ (dd, 1H, $J=2.4 \mathrm{~Hz}, J=3.9 \mathrm{~Hz}), 3.12(\mathrm{~d}, 1 \mathrm{H}, J=18.6 \mathrm{~Hz}), 2.32-2.20(\mathrm{~m}, 1 \mathrm{H}), 1.60-1.54(\mathrm{~m}, 1 \mathrm{H}), 1.37$ $(\mathrm{t}, 3 \mathrm{H}, J=7.2 \mathrm{~Hz}) ;{ }^{13} \mathrm{C} \mathrm{NMR}\left(75.0 \mathrm{MHz}, \mathrm{CDCl}_{3}\right) \delta 160.0,151.7,129.5,128.8,91.7,85.3$, 62.7, 61.9, 61.1, 55.3, 51.1, 43.5, 24.4, 14.3; IR (thin film) vmax 3485, 2981, 2935, 2877, 1724, 1596, 1468, 1384, 1258, 1210, 1142, 1102, $1018 \mathrm{~cm}^{-1}$; NOED (400 MHz, $\left.\mathrm{CDCl}_{3}\right)$ irrad. $\delta 6.06(\mathrm{H}-5)$ : 3.0\% enhancement at $\mathrm{H}-7$, irrad. $\delta 3.47(\mathrm{H}-3): 6.0 \%$ enhancement at $\mathrm{H}-2$. CIHRMS [M] ${ }^{+}$calculated for $\mathrm{C}_{14} \mathrm{H}_{16} \mathrm{BrNO}_{6}$ : 373.0161, found: [M+1] 374.0240, [M+2] $375.0291 ;[\alpha]_{\mathrm{D}}{ }^{22}=-60.4\left(\mathrm{c}=0.014, \mathrm{CHCl}_{3}\right)$; syn-spiroisoxazoline ester (13): M.p. 90-93 ${ }^{0} \mathrm{C} .{ }^{1} \mathrm{H}$ NMR $\left(300 \mathrm{MHz}, \mathrm{CDCl}_{3}\right) \delta 6.12(\mathrm{~d}, 1 \mathrm{H}, J=2.4 \mathrm{~Hz}), 4.37$ (q, 2H, $\left.J=7.2 \mathrm{~Hz}\right)$, 4.26-4.09 (m, 4H), $4.07(\mathrm{~d}, 1 \mathrm{H}, J=3.9 \mathrm{~Hz}), 3.49(\mathrm{dd}, 1 \mathrm{H}, J=2.7 \mathrm{~Hz}, J=3.9 \mathrm{~Hz}), 3.30(\mathrm{~d}, 1 \mathrm{H}$, $J=18.3 \mathrm{~Hz}), 3.23(\mathrm{~d}, 1 \mathrm{H}, J=18.6 \mathrm{~Hz}), 2.26-2.14(\mathrm{~m}, 1 \mathrm{H}), 1.67-1.58(\mathrm{~m}, 1 \mathrm{H}), 1.37(\mathrm{t}, 3 \mathrm{H}, J$ $=7.2 \mathrm{~Hz}) ;{ }^{13} \mathrm{C} \mathrm{NMR}\left(75.0 \mathrm{MHz}, \mathrm{CDCl}_{3}\right) \delta 160.0,151.1,129.7,126.0,92.6,87.4,62.7$, 61.7, 61.2, 54.3, 49.4, 43.0, 24.5, 14.3; IR (thin film) vmax 3460, 2980, 2880, 2721, 1723, $1654,1595,1469,1433,1382,1256,1142,1017 \mathrm{~cm}^{-1} ;$ NOED $\left(400 \mathrm{MHz}, \mathrm{CDCl}_{3}\right)$ irrad. $\delta$ 6.12 (H-5): $1.0 \%$ enhancement at $\mathrm{H}-7$, irrad. $\delta 3.49$ (H-3): $6.7 \%$ enhancement at $\mathrm{H}-2,2.6 \%$

S3 Lei, X.; Johnson, R. P.; Porco, J. A Jr. Angew. Chem. Int. Ed. 2003, 42, 3913. 
enhancement at H-8. CIHRMS [M] $]^{+}$calculated for $\mathrm{C}_{14} \mathrm{H}_{16} \mathrm{BrNO}_{6}$ : 373.0161, found: [M+1] $374.0238 ;[\alpha]_{\mathrm{D}}^{22}=+82.4\left(\mathrm{c}=0.041, \mathrm{CHCl}_{3}\right)$

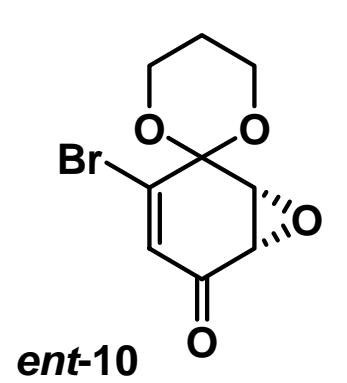

Quinone monoepoxide (ent-10): $\mathrm{Ph}_{3} \mathrm{COOH}(4.14 \mathrm{~g}, 14.99$ $\mathrm{mmol})$ was dissolved in $20 \mathrm{~mL}$ toluene, and $(12.8 \mathrm{~mL}, 12.8 \mathrm{mmol})$ of $1.0 \mathrm{M}$ NaHMDS in THF was added at rt. After $10 \mathrm{~min},(1.0 \mathrm{~g}, 4.28$ mmol) $D$-DIPT in $5 \mathrm{~mL}$ toluene was added, the reaction mixture was stirred for $10 \mathrm{~min}$ at $\mathrm{rt}$, and then cooled to $-78^{\circ} \mathrm{C}$. Cyclic ketal $9(1.05$ $\mathrm{g}, 4.28 \mathrm{mmol}$ ) in $10 \mathrm{~mL}$ toluene was added dropwise and the reaction mixture was stirred at $-50{ }^{\circ} \mathrm{C}$ for $15 \mathrm{~h}$. The reaction was quenched with water and extracted with EtOAc. The combined organic layers were washed with brine, dried over $\mathrm{MgSO}_{4}$, filtered, and concentrated in vacuo. Purification on silica gel $\left(20 \% \mathrm{Et}_{2} \mathrm{O}\right.$ in hexane to $40 \% \mathrm{Et}_{2} \mathrm{O}$ in hexane) provided $0.92 \mathrm{~g}(3.52 \mathrm{mmol}, 82 \%)$ of quinone monoepoxide ent-10 as a white solid which was crystallized from 20\% EtOAc in hexane at $-20{ }^{\circ} \mathrm{C}$. M.p. $145-146{ }^{\circ} \mathrm{C} ;{ }^{1} \mathrm{H}$ NMR $\left(400 \mathrm{MHz}, \mathrm{CDCl}_{3}\right) 6.43(\mathrm{~d}, 1 \mathrm{H}, J=2.0 \mathrm{~Hz}), 4.32$ $(\mathrm{m}, 2 \mathrm{H}), 4.27(\mathrm{~d}, 1 \mathrm{H}, J=3.6 \mathrm{~Hz}), 4.20(\mathrm{~m}, 2 \mathrm{H}), 3.54(\mathrm{q}, 1 \mathrm{H}, J=8.0 \mathrm{~Hz}), 2.28(\mathrm{~m}, 1 \mathrm{H}), 1.68$ $(\mathrm{m}, 1 \mathrm{H}) ;{ }^{13} \mathrm{C}$ NMR $\left(75.0 \mathrm{MHz}, \mathrm{CDCl}_{3}\right) \delta 189.1,146.2,130.0,92.2,61.4,61.2,52.8,51.5$, 24.1; IR (thin film) vmax 3058, 2962, 2873, 1690,1623,1290, 1246, 1138, $1105 \mathrm{~cm}^{-1}$; CIHRMS $[\mathrm{M}+\mathrm{H}]^{+}$calculated for $\mathrm{C}_{9} \mathrm{H}_{10} \mathrm{BrO}_{4}: 260.9762$, found: $260.9726 ;[\alpha]_{\mathrm{D}}^{23}=-184(\mathrm{c}=$ $0.020 \mathrm{CHCl}_{3}$ ). The ee was determined to be $98.4 \%$ after crystallization using a Regis chiral HPLC column $(\mathrm{R}, \mathrm{R})$ whelk-O1 $(25 \mathrm{~cm} \times 4.0 \mathrm{~mm}(15 \%$ isopropanol in hexane, flow rate $0.5 \mathrm{~mL} / \mathrm{min}$; retention time $25.28 \mathrm{~min}$ (minor enantiomer) and $29.87 \mathrm{~min}$ (major enantiomer)).

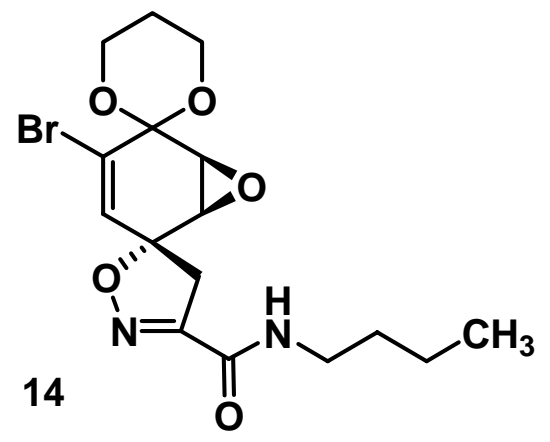

Monoamide (14): To 2-hydroxypyridine (28 mg, 0.29 $\mathrm{mmol})$ in $2 \mathrm{~mL}$ of toluene was added $\mathrm{Zr}\left(\mathrm{O}^{\mathrm{t}} \mathrm{Bu}\right)_{4}(21 \mu \mathrm{L}$, $0.05 \mathrm{mmol})$ followed by $n$-butylamine $(32 \mu \mathrm{L}, 0.32$ $\mathrm{mmol})$ at rt. The anti-spiroisoxazoline ester $12(100 \mathrm{mg}$, $0.26 \mathrm{mmol}$ ) was dissolved in $1 \mathrm{~mL}$ toluene and added to the reaction mixture which was heated to $60{ }^{\circ} \mathrm{C}$ and stirred for $8 \mathrm{~h}$. The mixture was cooled to rt and filtered thru a pad of Celite. The filtrate was evaporated to dryness and directly subjected to purification on silica gel $(40 \%$ EtOAc in petroleum ether) to afford $80 \mathrm{mg}(0.2$ mmol, $75 \%)$ of 14 as a yellow oil. ${ }^{1} \mathrm{H}$ NMR $(300 \mathrm{MHz}$, $\left.\left(\mathrm{CD}_{3}\right)_{2} \mathrm{CO}\right) \delta 7.61(\mathrm{~b}, 1 \mathrm{H}), 6.26(\mathrm{~d}, 1 \mathrm{H}, J=2.4 \mathrm{~Hz}), 4.45-4.29(\mathrm{~m}, 2 \mathrm{H}), 4.39(\mathrm{~d}, 1 \mathrm{H}, J=3.9$ $\mathrm{Hz}), 4.05-3.95(\mathrm{~m}, 2 \mathrm{H}), 3.56(\mathrm{dd}, 1 \mathrm{H}, J=2.4 \mathrm{~Hz}, J=3.9 \mathrm{~Hz}), 3.51(\mathrm{~d}, 1 \mathrm{H}, J=17.7 \mathrm{~Hz})$, 3.34-3.27 (m, 3H), 2.13-2.10 (m, 1H), 1.62-1.49 (m, 3H), 1.41-1.29 (m, 2H), $0.93(\mathrm{t}, 3 \mathrm{H}, \mathrm{J}=$ $7.2 \mathrm{~Hz}) ;{ }^{13} \mathrm{C}$ NMR $\left(75.0 \mathrm{MHz},\left(\mathrm{CD}_{3}\right)_{2} \mathrm{CO}\right) \delta 159.0,154.5,129.5,129.0,91.9,84.6,61.4$, $60.5,54.8,50.6,43.4,38.9,31.6,24.5,19.9,13.3$; IR (thin film) vmax 3335, 2959, 2931, $2873,1664,1599,1539,1466,1434,1385,1142,1113,1017,1046 \mathrm{~cm}^{-1}$; EIHRMS calculated for $\mathrm{C}_{16} \mathrm{H}_{21} \mathrm{BrN}_{2} \mathrm{O}_{5}[\mathrm{M}+\mathrm{H}]^{+}: 401.0713$, found: 401.0708; $[\alpha]_{\mathrm{D}}^{22}=-72.3(\mathrm{c}=$ $\left.0.028, \mathrm{CHCl}_{3}\right)$. 


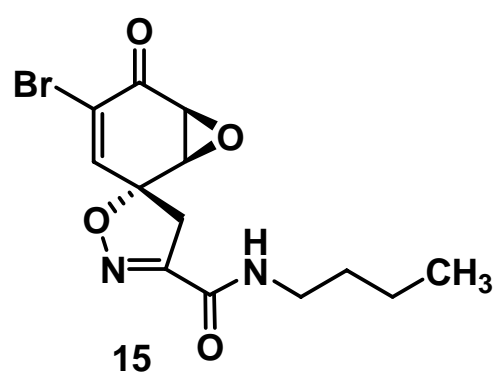

Monoamide (15): Monoamide 14 (30 $\mathrm{mg}, 0.07 \mathrm{mmol})$ was dissolved in $1 \mathrm{~mL}$ of $\mathrm{HPLC}$ grade $\mathrm{CH}_{3} \mathrm{CN}$ and $5 \mathrm{~mL}$ of $\mathrm{CH}_{2} \mathrm{Cl}_{2} .0 .8 \mathrm{~mL}$ of $\mathrm{HF}\left(48 \%\right.$ in $\left.\mathrm{H}_{2} \mathrm{O}\right)$ was added at $0{ }^{\circ} \mathrm{C}$. The mixture was stirred at $\mathrm{rt}$ for $6 \mathrm{~h}$ before being quenched with satd. aqueous $\mathrm{NaHCO}_{3}$. The mixture was extracted with EtOAc, the organic extracts were combined, washed with brine, dried over $\mathrm{MgSO}_{4}$, filtered, and concentrated in vacuo. Purification on silica gel (40\% EtOAc in hexane) provided $20 \mathrm{mg}(0.06 \mathrm{mmol}, 78 \%)$ of $\mathbf{1 5}$ as a white solid. M.p. 178$180{ }^{\circ} \mathrm{C} ;{ }^{1} \mathrm{H}$ NMR $\left(400 \mathrm{MHz}, \mathrm{CDCl}_{3}\right) \delta 6.89(\mathrm{~d}, 1 \mathrm{H}, J=2.4$ $\mathrm{Hz}), 6.58(\mathrm{~b}, 1 \mathrm{H}), 3.77-3.73(\mathrm{~m}, 3 \mathrm{H}), 3.43(\mathrm{~d}, 1 \mathrm{H}, J=18.8 \mathrm{~Hz}), 3.37$ (q, $2 \mathrm{H}, J=7.2 \mathrm{~Hz})$, 1.59-1.50 (m, 2H), 1.41-1.31 (m, 2H), 0.94(t, 3H, $J=7.2 \mathrm{~Hz}) ;{ }^{13} \mathrm{C}$ NMR $(75.0 \mathrm{MHz}$, $\left.\mathrm{CDCl}_{3}\right) \delta 185.0,158.3,154.3,141.6,125.1,85.1,57.3,53.5,44.0,39.6,31.6,20.2,13.9$; IR (thin film) vmax 3304, 2961, 2929, 2861, 1701, 1649, 1605, 1549, 1434, 1344, 1274, 1099, $1035 \mathrm{~cm}^{-1}$; EIHRMS $[\mathrm{M}]^{+}$calculated for $\mathrm{C}_{13} \mathrm{H}_{15} \mathrm{BrN}_{2} \mathrm{O}_{4}: 342.0215$, found: $342.0181 ;[\alpha]_{\mathrm{D}}{ }^{22}$ $=-106\left(\mathrm{c}=0.004, \mathrm{CHCl}_{3}\right)$.

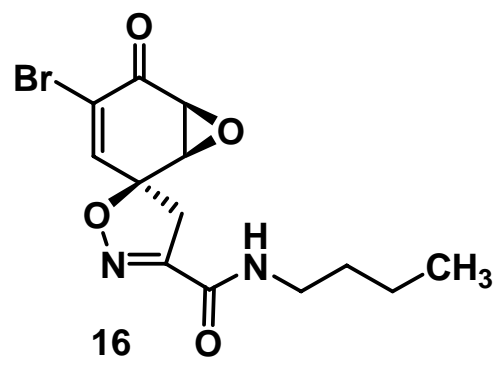

Monoamide (16): Prepared in 69\% yield from 22 as a yellow oil according to the procedure reported for $15 .{ }^{1} \mathrm{H}$ NMR $\left(400 \mathrm{MHz},\left(\mathrm{CD}_{3}\right)_{2} \mathrm{CO}\right) \delta 7.68(\mathrm{~b}, 1 \mathrm{H}), 7.27(\mathrm{~d}, 1 \mathrm{H}, J$ $=3.2 \mathrm{~Hz}), 4.16(\mathrm{dd}, 1 \mathrm{H}, J=2.4,3.6 \mathrm{~Hz}), 3.78(\mathrm{~d}, 1 \mathrm{H}, J=$ $3.6 \mathrm{~Hz}), 3.70(\mathrm{~d}, 1 \mathrm{H}, J=18.4 \mathrm{~Hz}), 3.55(\mathrm{~d}, 1 \mathrm{H}, J=18.4$ $\mathrm{Hz}), 3.31(\mathrm{q}, 2 \mathrm{H}, J=7.2 \mathrm{~Hz}), 1.60-1.50(\mathrm{~m}, 2 \mathrm{H}), 1.40-1.32$ $(\mathrm{m}, 2 \mathrm{H}), 0.93(\mathrm{t}, 3 \mathrm{H}, J=7.2 \mathrm{~Hz}) ;{ }^{13} \mathrm{C}$ NMR $(75.0 \mathrm{MHz}$, $\left.\left(\mathrm{CD}_{3}\right)_{2} \mathrm{CO}\right) \delta 185.8,158.7,154.7,144.2,120.1,86.1,55.6$, 52.1, 43.3, 38.9, 31.6, 19.9, 13.3; IR (thin film) vmax 3307, $3043,2957,2932,2872,1669,1655,1609,1545,1434$, 1337, 1271, $1019 \mathrm{~cm}^{-1}$; EIHRMS [M] calculated for $\mathrm{C}_{13} \mathrm{H}_{15} \mathrm{BrN}_{2} \mathrm{O}_{4}$ : 342.0215 , found: $342.0201 ;[\alpha]_{\mathrm{D}}^{22}=-10.9\left(\mathrm{c}=0.004, \mathrm{CHCl}_{3}\right)$. 


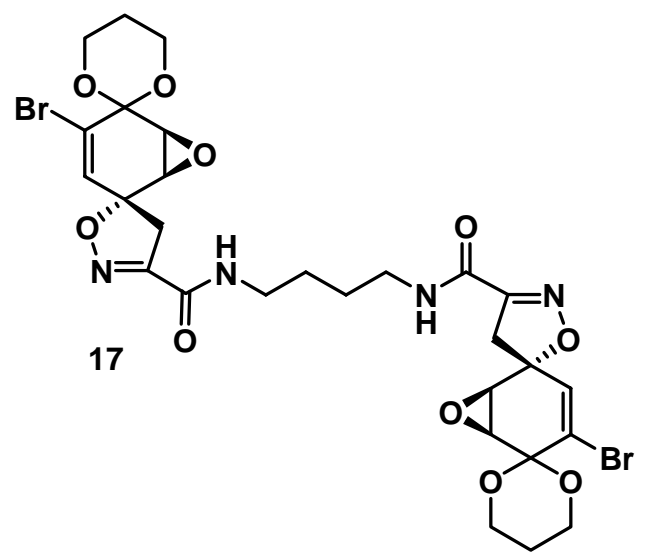

Bis-ketal (17): To 2-hydroxypyridine (12 mg, $0.13 \mathrm{mmol})$ in $1 \mathrm{~mL}$ toluene was added $\mathrm{Zr}\left(\mathrm{O}^{\mathrm{t}} \mathrm{Bu}\right)_{4}$ (10 $\mu \mathrm{L}, 0.02 \mathrm{mmol}), \mathrm{Zn}(\mathrm{OTf})_{2}(9 \mathrm{mg}, 0.02 \mathrm{mmol})$, followed by 1,4 diamino butane $(7 \mu \mathrm{L}, 0.07 \mathrm{mmol})$ at rt. Anti-spiroisoxazoline ester $12(45 \mathrm{mg}, 0.12$ $\mathrm{mmol})$ was dissolved in toluene $(0.05 \mathrm{~mL}, 0.1 \mathrm{M})$ and added to the reaction mixture which was heated to $60^{\circ} \mathrm{C}$ and stirred for $4 \mathrm{~h}$. The mixture was cooled to $\mathrm{rt}$ and filtered through a pad of Celite. The filtrate was evaporated to dryness and directly subjected to purification on neutral alumina $(\mathrm{pH}$ 9-10) $\left(\mathrm{CH}_{2} \mathrm{Cl}_{2} / \mathrm{EtOAc} / \mathrm{MeOH}=2: 1: 0.2\right)$ to provide $35 \mathrm{mg}$ (0.05 mmol, 78\%) of bis-amide $\mathbf{1 7}$ as a white solid.

M.p. 211-212 ${ }^{\circ} \mathrm{C} ;{ }^{1} \mathrm{H}$ NMR $\left(400 \mathrm{MHz},\left(\mathrm{CD}_{3}\right)_{2} \mathrm{CO}\right) \delta 7.69$ (bt, $\left.2 \mathrm{H}\right), 6.26(\mathrm{~d}, 2 \mathrm{H}, J=2.4 \mathrm{~Hz})$, $4.40(\mathrm{~d}, 2 \mathrm{H}, J=4.0 \mathrm{~Hz}), 4.44-4.31(\mathrm{~m}, 4 \mathrm{H}), 4.05-3.96(\mathrm{~m}, 4 \mathrm{H}), 3.57$ (dd, $2 \mathrm{H}, J=2.4,4.0$ $\mathrm{Hz}), 3.50(\mathrm{~d}, 2 \mathrm{H}, J=18.4 \mathrm{~Hz}), 3.34-3.29(\mathrm{~m}, 6 \mathrm{H}), 2.17-2.07(\mathrm{~m}, 2 \mathrm{H}), 1.92-1.83(\mathrm{~m}, 2 \mathrm{H}),{ }^{13} \mathrm{C}$ NMR $\left(75.5 \mathrm{MHz},\left(\mathrm{CD}_{3}\right)_{2} \mathrm{CO}\right) \delta 159.1,154.5,129.5,129.0,91.9,84.6,61.4,60.5,54.8,50.6$, 43.4, 38.8, 26.8, 24.5; IR (thin film) vmax 1732, 1654, 1539, 1456, 1384, 1260, 1141, 1101, $1016 \mathrm{~cm}^{-1}$; EIHRMS calculated for $\mathrm{C}_{28} \mathrm{H}_{32} \mathrm{Br}_{2} \mathrm{~N}_{4} \mathrm{O}_{10}[\mathrm{M}]^{+}: 742.0485$, found: $742.9663[\alpha]_{\mathrm{D}}{ }^{22}$ $=-93.3\left(\mathrm{c}=0.003, \mathrm{CHCl}_{3}\right)$.

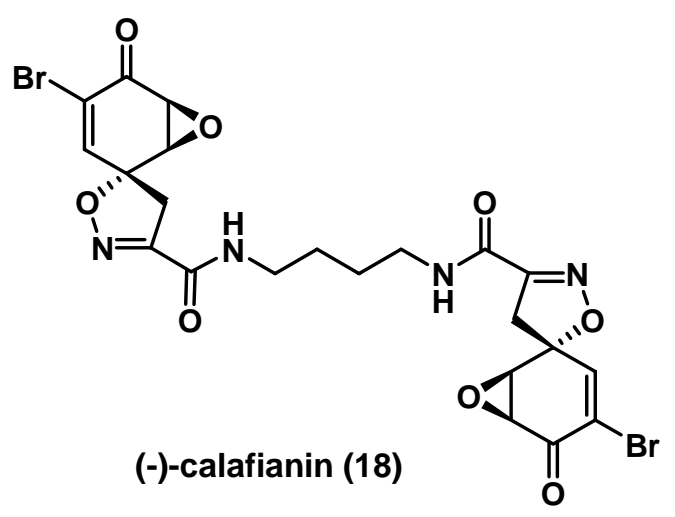

(-)-Calafianin (18): Bis-ketal $17(25 \mathrm{mg}$, $0.03 \mathrm{mmol}$ ) was dissolved in $0.5 \mathrm{~mL}$ of HPLC grade $\mathrm{CH}_{3} \mathrm{CN}$ and $2.5 \mathrm{~mL} \mathrm{CH}_{2} \mathrm{Cl}_{2}$. $0.5 \mathrm{~mL} \mathrm{HF}$ $\left(48 \%\right.$ in $\left.\mathrm{H}_{2} \mathrm{O}\right)$ was added at $0^{\circ} \mathrm{C}$. The mixture was stirred at $\mathrm{rt}$ for $12 \mathrm{~h}$ before being quenched with satd. aqueous $\mathrm{NaHCO}_{3}$. The mixture was extracted with a solvent mixture of $\mathrm{CHCl}_{3} / \mathrm{iPrOH}$ (2:1); the organic extracts were combined, dried over $\mathrm{Na}_{2} \mathrm{SO}_{4}$, filtered, and concentrated in vacuo. Purification on silica gel, neutral alumina, or using preparative HPLC led to decomposition. Hence a crude yield of $18 \mathrm{mg}(0.02 \mathrm{mmol}, 90 \%)$ of $\mathbf{1 8}$ was obtained and further characterized as a

white solid. ${ }^{1} \mathrm{H}$ NMR (300 MHz, DMSO-d $\left.{ }_{6}\right) \delta 8.63(\mathrm{t}, 2 \mathrm{H}, J=5.4 \mathrm{~Hz}), 7.47$ (d, $2 \mathrm{H}, J=2.4$ $\mathrm{Hz}$ ), 4.12 (dd, 2H, $J=2.4,3.6 \mathrm{~Hz}$ ), 3.93 (d, 2H, $J=3.6 \mathrm{~Hz}$ ), 3.69 (d, 4H, $J=17.4 \mathrm{~Hz}$ ), 3.61 $(\mathrm{d}, 4 \mathrm{H}, J=17.4 \mathrm{~Hz}), 3.17(\mathrm{br}, 4 \mathrm{H}), 1.47(\mathrm{br}, 4 \mathrm{H}) ;{ }^{13} \mathrm{C}$ NMR $\left(75.0 \mathrm{MHz}, \mathrm{DMSO}-\mathrm{d}_{6}\right) \delta$ $186.6,158.9,155.5,144.3,123.4,84.6,57.5,53.6,44.2,39.2,26.9$; IR (thin film) vmax $3355,2924,2854,1726,1662,1602,1543,1457,1268,1121,1074 \mathrm{~cm}^{-1}$; FABMS calculated for $\mathrm{C}_{22} \mathrm{H}_{20} \mathrm{Br}_{2} \mathrm{~N}_{4} \mathrm{O}_{8} \mathrm{Na}[\mathrm{M}+\mathrm{Na}]^{+}:$648.9546, found: 648 ; $[\alpha]_{\mathrm{D}}{ }^{22}=-14.1(\mathrm{c}=0.001$, EtOH). CD spectra; $\lambda$, (rotation in millidegrees) 320 (-4.6), $270(-23.4), 233$ (-44.0), 205 (+27.6). 
(+)-Epi-calafianin (19): Prepared from 23 in $92 \%$ yield as a white solid according to the procedure reported for (-)-calafianin 18. ${ }^{1} \mathrm{H}$ NMR $\left(300 \mathrm{MHz}, \mathrm{DMSO}_{-} \mathrm{d}_{6}\right) \delta 8.60(\mathrm{t}, 2 \mathrm{H}, J=$ $5.4 \mathrm{~Hz}), 7.28(\mathrm{~d}, 2 \mathrm{H}, J=2.4 \mathrm{~Hz}), 4.10(\mathrm{~m}, 2 \mathrm{H}), 3.79(\mathrm{dd}, 2 \mathrm{H}, J=2.4,3.6 \mathrm{~Hz}), 3.58(\mathrm{~d}, 2 \mathrm{H}, J$ $=17.4 \mathrm{~Hz}), 3.42(\mathrm{~d}, 2 \mathrm{H}, J=17.4 \mathrm{~Hz}), 3.13(\mathrm{br}, 4 \mathrm{H}), 1.45(\mathrm{br}, 4 \mathrm{H}) ;{ }^{13} \mathrm{C}$ NMR $(75.0 \mathrm{MHz}$, DMSO-d $_{6}$ ) $\delta 186.4,159.1,155.3,144.8,120.3,86.1,55.7,52.4,43.9,39.1,26.9$; IR (thin film) vmax 3308, 2921, 2851, 1700, 1654, 1608, 1556, 1541, 1456, 1262, 1215, $1019 \mathrm{~cm}^{-1}$; ESI-HRMS calculated for $\mathrm{C}_{22} \mathrm{H}_{20} \mathrm{Br}_{2} \mathrm{~N}_{4} \mathrm{O}_{8} \mathrm{Na}[\mathrm{M}+\mathrm{Na}]^{+}: 648.9546$, found: $648.9565 ; \quad[\alpha]_{\mathrm{D}}{ }^{22}$ $=+1.8(\mathrm{c}=0.004, \mathrm{EtOH}) . \mathrm{CD}$ spectra; $\lambda$, (rotation in millidegrees) $204(+15.9)$

(+)-Calafianin (3): $\left[\alpha_{D}\right]^{22}=+10.8(c=0.006, E t O H)$. The value for the specific rotation of natural calafianin was not determined in the isolation report ${ }^{4}$ due to solubility issues. However, a positive sign was assigned in ethanol as solvent. CD spectra; $\lambda$, (rotation in millidegrees) 320 (0.12), 270 (12.7), 233 (30.0), 206 (-11.0), 205 (-6.0).

(-)-Epi-calafianin (20): $[\alpha]_{\mathrm{D}}^{22}=-5.3(\mathrm{c}=0.004, \mathrm{EtOH}) . \mathrm{CD}$ spectra; $\lambda$, (rotation in millidegrees) 204 (-13.9)

\footnotetext{
${ }^{4}$ Encarnacion, R. D.; Sandoval, E.; Mamstrom, J.; Christophersen, C. J. Nat. Prod. 2000, 63, 874
} 


\section{CD curves for natural $(+)$-calafianin, and synthetic $(-)$ calafianin, $(+)$-epi-}

calafianin, and (-)-calafianin

In a typical experiment for recording CD spectra, a $1 \mathrm{mM}$ solution of (-)-calafianin 18 was prepared in freshly distilled and degassed $\mathrm{EtOH}$. UV absorption was recorded to keep the absorbance below 1.0 for the range of wavelength to be recorded ( $320 \mathrm{~nm}$ to $205 \mathrm{~nm}$ ).

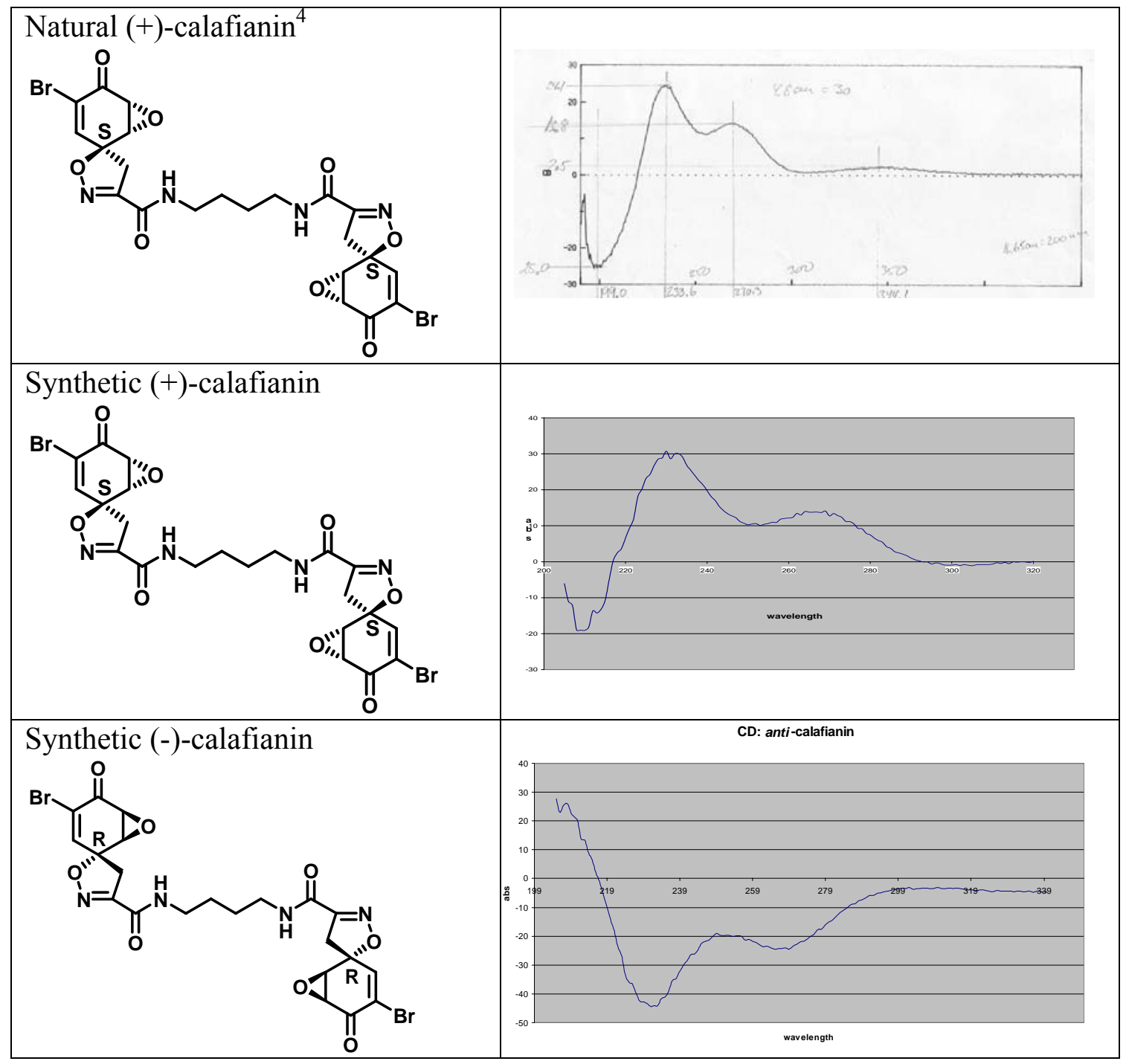




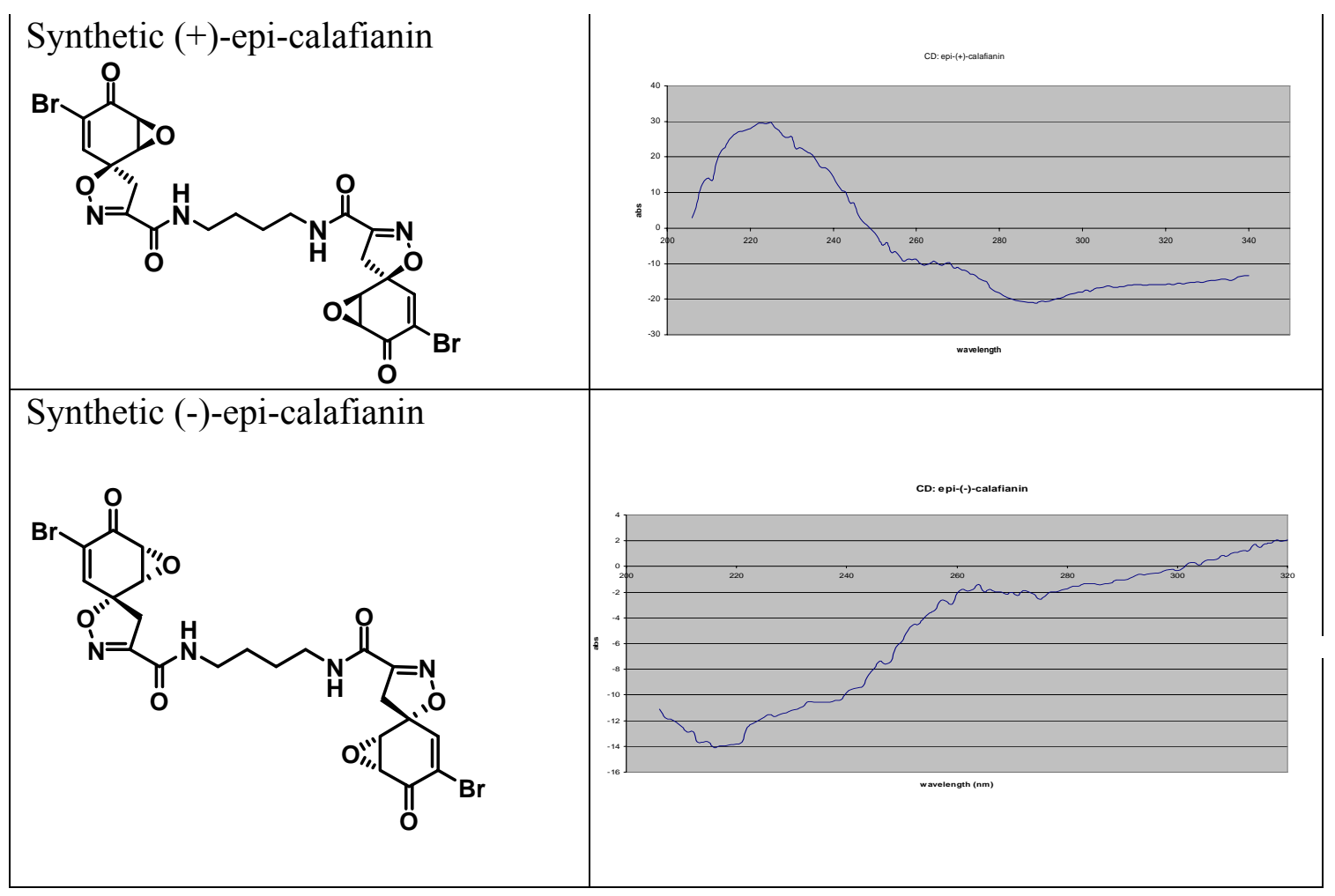

NMR data (in DMSO-d $\mathrm{d}_{6}$ ) comparison of natural and synthetic calafianin

\begin{tabular}{|c|c|c|c|c|c|}
\hline No & Natural (400 MHz) & Synthetic (300 MHz) & COSY (400 MHz) & Natural (100 MHz) & Synthetic (75.0 MHz) \\
\hline $1,1^{\prime}$ & $4.13(\mathrm{dd}, 2.6,3.7)$ & $4.12(\mathrm{dd}, 2.4,3.6)$ & $\mathrm{H} 5, \mathrm{H} 5^{\prime}, \mathrm{H} 2, \mathrm{H} 2^{\prime}$ & 56.9 & 57.8 \\
\hline 2,2 & $3.93(\mathrm{~d}, 3.5)$ & $3.93(\mathrm{~d}, 3.6)$ & $\mathrm{H} 1, \mathrm{H} 1{ }^{\prime}$ & 53.0 & 53.6 \\
\hline $3,3^{\prime}$ & & & & 186.0 & 186.6 \\
\hline $4,4^{\prime}$ & & & & 122.8 & 123.4 \\
\hline 5,5 & $7.49(\mathrm{~d}, 2.6)$ & $7.47(\mathrm{~d}, 2.4)$ & $\mathrm{H} 1, \mathrm{H} 1{ }^{\prime}$ & 143.7 & 144.3 \\
\hline $6,6^{\prime}$ & & & & 84.0 & 84.6 \\
\hline 7,7 & $\begin{array}{l}3.68(\mathrm{~d}, 17.8) \\
3.61(\mathrm{~d}, 17.9)\end{array}$ & $\begin{array}{l}3.69(\mathrm{~d}, 17.4) \\
3.61(\mathrm{~d}, 17.4)\end{array}$ & & 43.6 & 44.2 \\
\hline $8,8^{\prime}$ & & & & 154.9 & 155.5 \\
\hline $9,9^{\prime}$ & & & & 158.3 & 158.9 \\
\hline $10,10^{\prime}$ & 3.19 (br) & 3.17 (br) & $\begin{array}{l}\mathrm{H} 11, \mathrm{H} 11 \\
\mathrm{NH}, \mathrm{NH}\end{array}$ & 38.6 & 39.2 \\
\hline $11,11^{\prime}$ & 1.50 (br) & 1.47 (br) & H10, H10' & 26.4 & 26.9 \\
\hline $\mathrm{NH}$ & $8.63(\mathrm{t}, 5.7)$ & $8.63(\mathrm{t}, 5.4)$ & $\mathrm{H} 10, \mathrm{H} 10^{\prime}$ & & \\
\hline
\end{tabular}


${ }^{1}$ H-NMR (400 MHz, DMSO-d 6 )

Natural (+)-calafianin
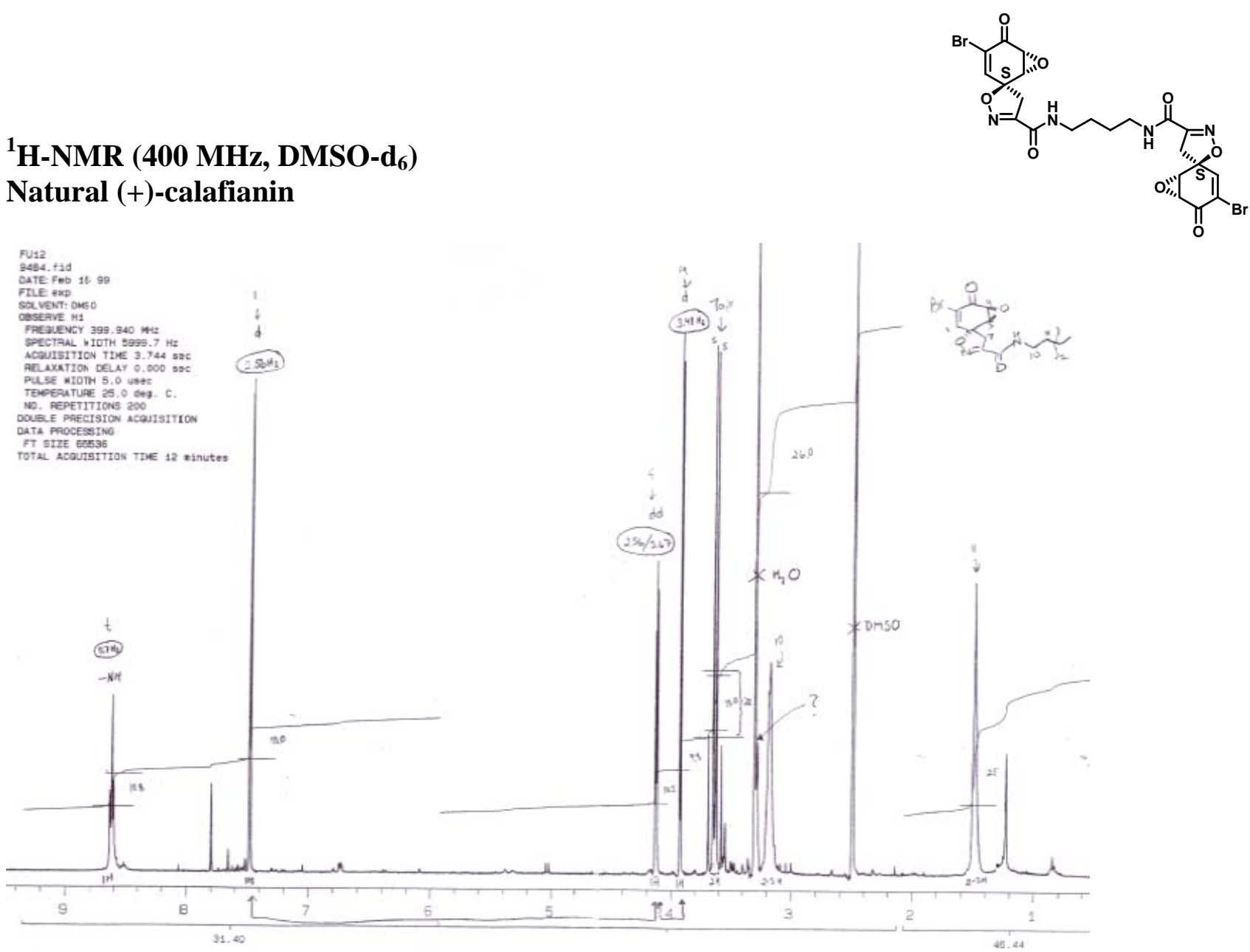

${ }^{1}$ H-NMR (300 MHz, DMSO-d ${ }_{6}$ )

Synthetic (+)-calafianin

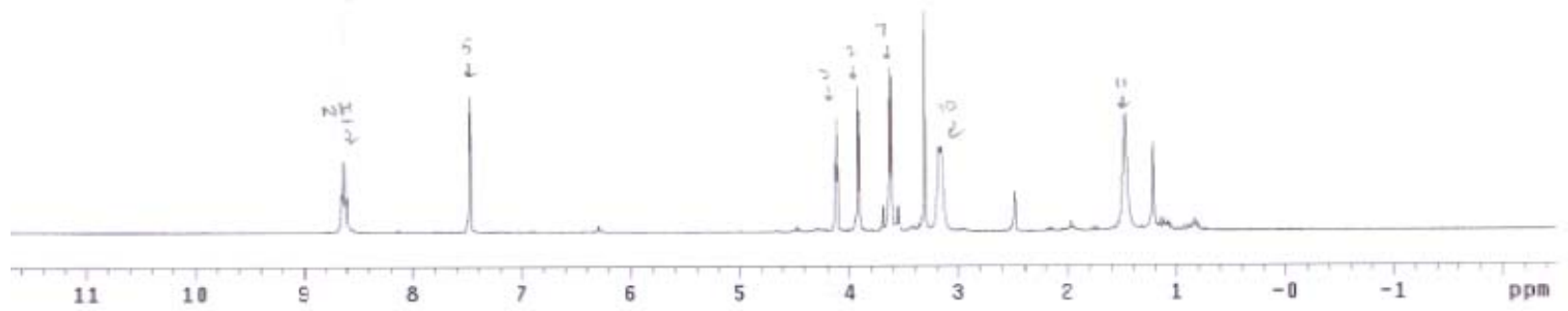


COSY (400 MHz, DMSO-d ${ }_{6}$ )
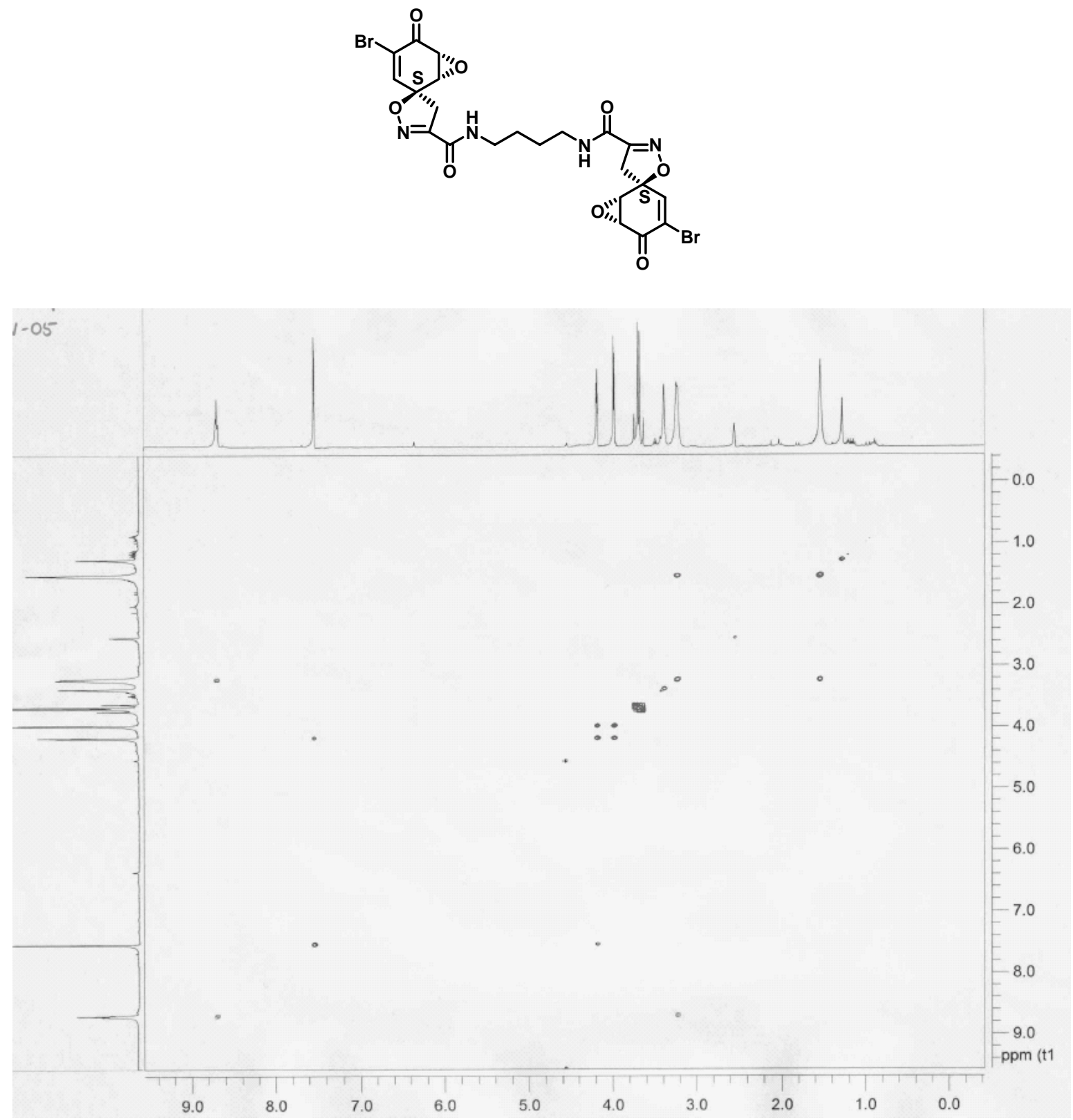
${ }^{13}$ C-NMR (100 MHz, DMSO-d ${ }_{6}$ )

Natural (+)-calafianin

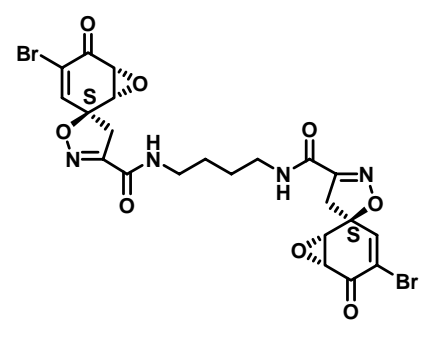

574 102

$25000.0 \mathrm{~kg}$
E $1.189 \mathrm{sec}$

.0 deac $c$.

$\$ 28096$.

twouscr an

1 Acoursstion

$11.0 \mathrm{~Hz}$

W. TINE 17.2 nour
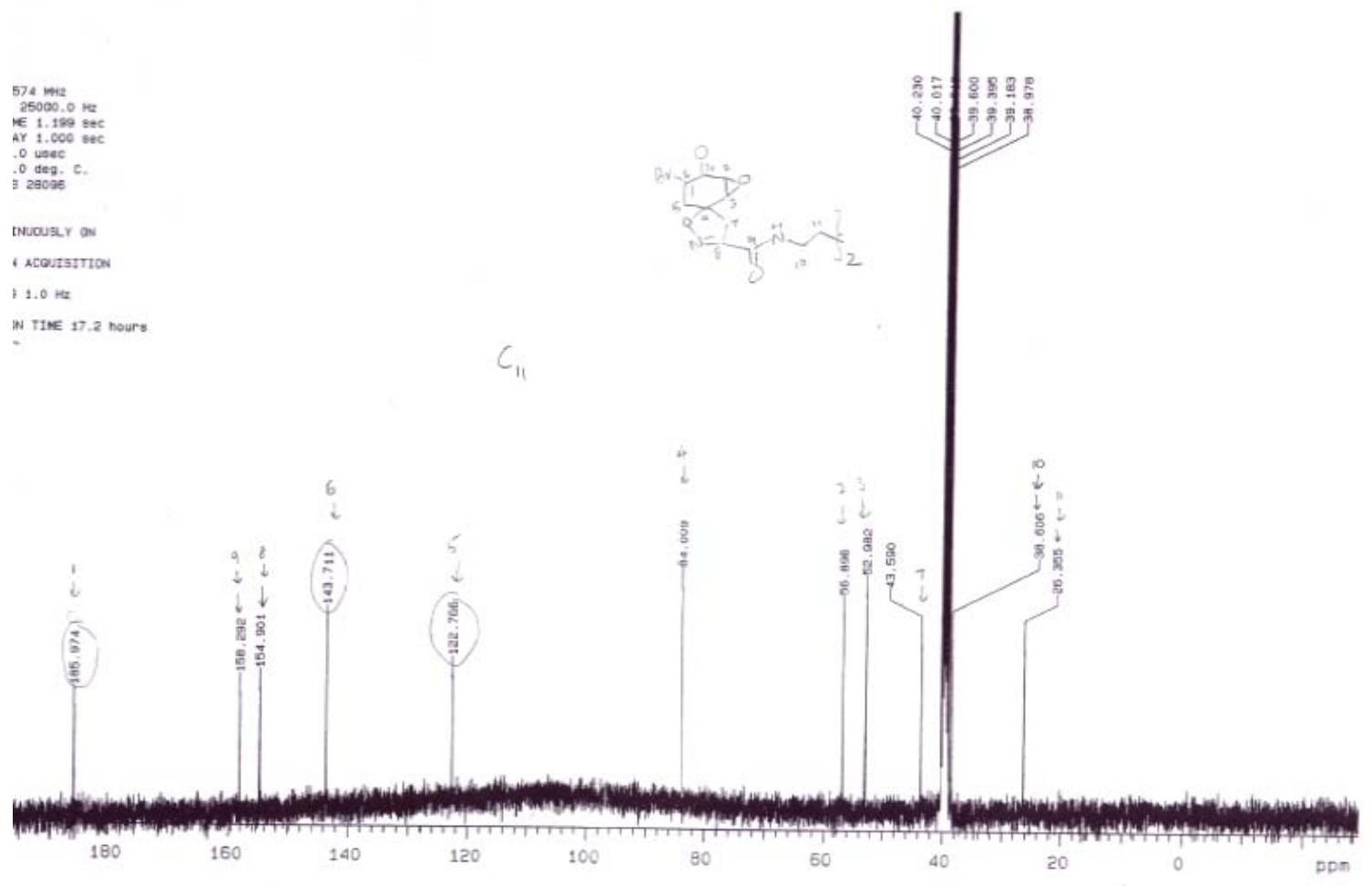

${ }^{13}$ C-NMR (75.0 MHz, DMSO-d ${ }_{6}$ ) Synthetic (+)-calafianin

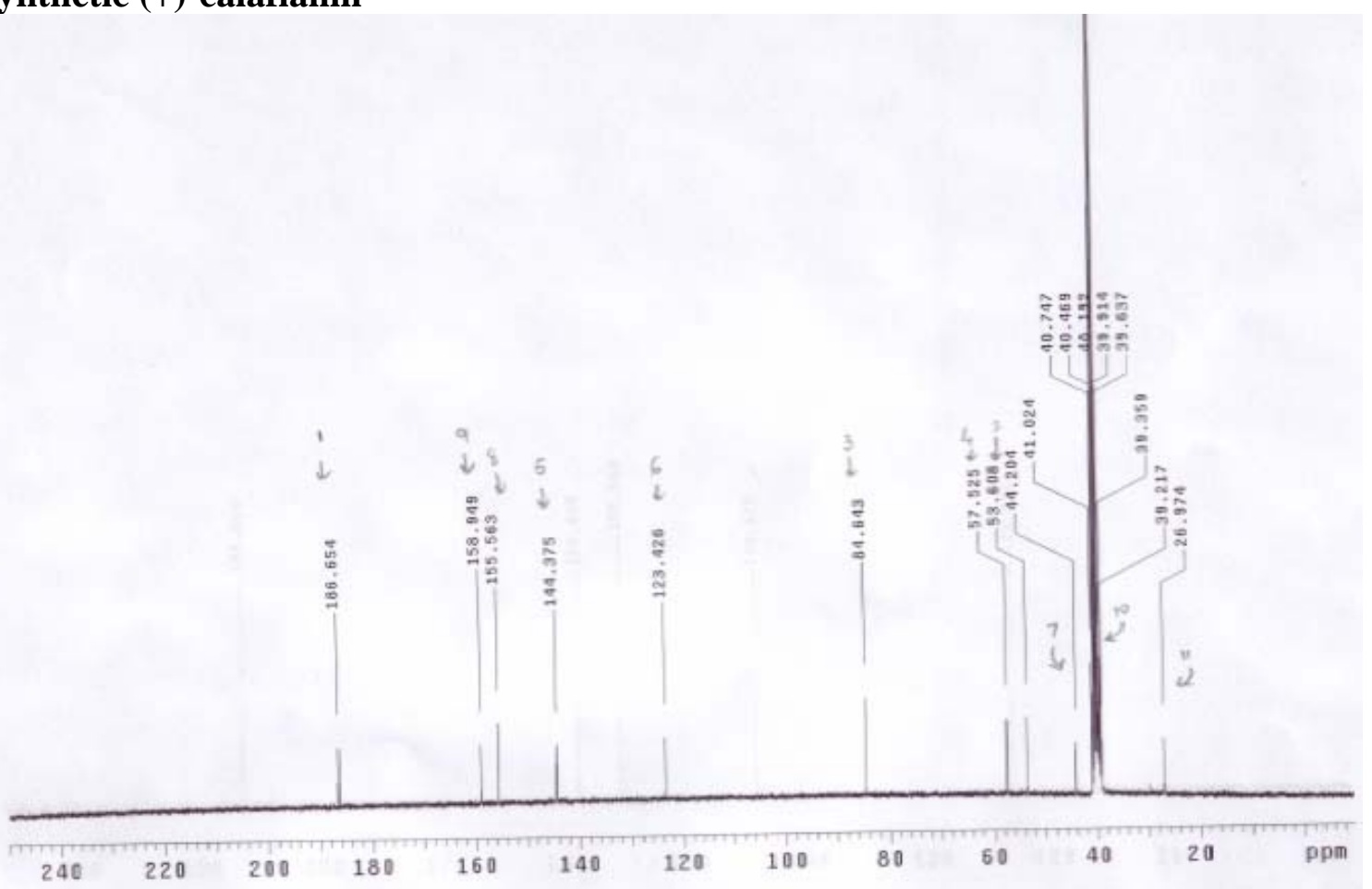




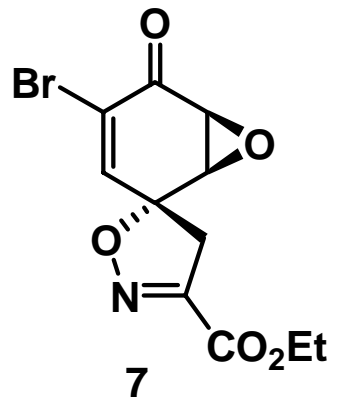

Spiroisoxazoline (7): Anti-spiroisoxazoline ester 12 (50 mg, 0.134 mmol) was dissolved in $1 \mathrm{~mL}$ of HPLC grade $\mathrm{CH}_{3} \mathrm{CN}$ and $5 \mathrm{~mL}$ of $\mathrm{CH}_{2} \mathrm{Cl}_{2} .0 .8 \mathrm{~mL} \mathrm{HF}\left(48 \%\right.$ in $\left.\mathrm{H}_{2} \mathrm{O}\right)$ was added at $0{ }^{\circ} \mathrm{C}$. The mixture was stirred at $\mathrm{rt}$ for $12 \mathrm{~h}$ before being quenched with satd. aqueous $\mathrm{NaHCO}_{3}$. The mixture was extracted with EtOAc, the organic extracts were combined, washed with brine, dried over $\mathrm{MgSO}_{4}$, filtered, and concentrated in vacuo. Purification on silica gel $(30 \%$ EtOAc in hexane) provided $41 \mathrm{mg}(0.13 \mathrm{mmol}, 98 \%)$ of 7 as a white solid. M.p. $119-121^{\circ} \mathrm{C} ;{ }^{1} \mathrm{H}$ NMR $\left(300 \mathrm{MHz}, \mathrm{CDCl}_{3}\right) \delta 6.90(\mathrm{~d}, 1 \mathrm{H}, J=2.1 \mathrm{~Hz})$, $4.41(\mathrm{q}, 2 \mathrm{H}, J=6.9 \mathrm{~Hz}), 3.77-3.76(\mathrm{~m}, 2 \mathrm{H}), 3.75(\mathrm{dd}, 1 \mathrm{H}, J=18.3$

$\mathrm{Hz}), 3.42(\mathrm{dd}, 1 \mathrm{H}, J=18.6 \mathrm{~Hz}), 1.40(\mathrm{t}, 3 \mathrm{H}, J=6.9 \mathrm{~Hz}) ;{ }^{13} \mathrm{C} \mathrm{NMR}\left(75.0 \mathrm{MHz}, \mathrm{CDCl}_{3}\right) \delta$ $184.9,159.6,151.9,141.4,125.4,85.5,63.1,57.4,53.6,44.1,14.3$; IR (solid) vmax 1733, 1714, 1698, 1608, 1369, 1333, 1256, 1151, $1021 \mathrm{~cm}^{-1}$; CIHRMS calculated for $\mathrm{C}_{11} \mathrm{H}_{10} \mathrm{BrNO}_{5}\left[\mathrm{M}^{+}\right]: 314.9722$, found: 314.9722; $[\alpha]_{\mathrm{D}}{ }^{22}=-253\left(\mathrm{c}=0.003, \mathrm{CHCl}_{3}\right)$.

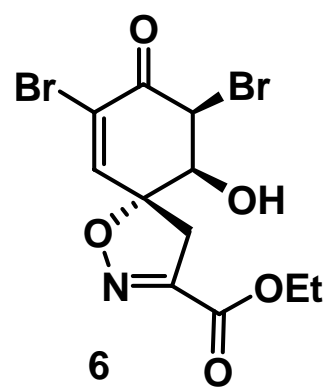

Bromohydrin (6): To $20 \mathrm{mg}(0.063 \mathrm{mmol})$ of 7 in $2 \mathrm{~mL}$ of dry $\mathrm{CH}_{3} \mathrm{CN}$ at $-10^{\circ} \mathrm{C}$ was added freshly prepared $\mathrm{MgBr}_{2} \cdot \mathrm{Et}_{2} \mathrm{O}$. The mixture was stirred for $2 \mathrm{~h}$, and the reaction was quenched with brine and extracted with EtOAc and dried with $\mathrm{Na}_{2} \mathrm{SO}_{4}$. The filtrate was concentrated, affording $25 \mathrm{mg}(0.063 \mathrm{mmol}, 100 \%)$ of 6 as a colorless oil and was chararacterized without further purification. ${ }^{1} \mathrm{H}$ NMR (400 MHz, $\left.\mathrm{CDCl}_{3}\right) \delta 7.38(\mathrm{~s}, 1 \mathrm{H}), 4.53(\mathrm{~d}, 1 \mathrm{H}, J=11.6 \mathrm{~Hz})$, 4.37-4.33 (m, 3H), $3.87(\mathrm{~d}, 1 \mathrm{H}, J=17.6 \mathrm{~Hz}), 3.11(\mathrm{~d}, 1 \mathrm{H}, J=18 \mathrm{~Hz})$, $1.36(\mathrm{t}, 3 \mathrm{H}, J=7.2 \mathrm{~Hz}) ;{ }^{13} \mathrm{C} \mathrm{NMR}\left(75.0 \mathrm{MHz}, \mathrm{CDCl}_{3}\right) \delta 182.2$, $159.7,151.5,147.7,123.1,91.1,74.5,62.9,55.6,37.5,14.3$; IR (thin film) vmax 3480, 2929, 1713, 1604, 1414, 1380, 1315, 1266, 1113, $1011 \mathrm{~cm}^{-1}$; CIHRMS calculated for $\mathrm{C}_{11} \mathrm{H}_{11} \mathrm{Br}_{2} \mathrm{NO}_{5}\left[\mathrm{M}^{+}\right]$: 394.9004, found: 394.9014; $[\alpha]_{\mathrm{D}}{ }^{22}=+10.0\left(\mathrm{c}=0.017, \mathrm{CHCl}_{3}\right)$.

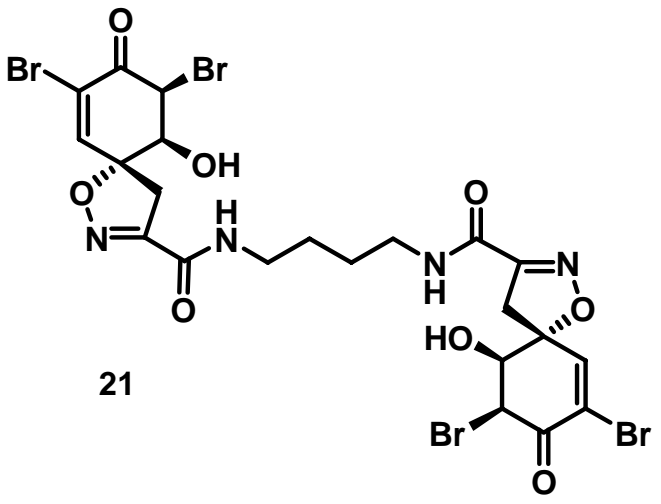

Bis-amide (21): To $15 \mathrm{mg}(0.023 \mathrm{mmol})$ of (-)-calafianin (18) in $1.5 \mathrm{~mL}$ of dry $\mathrm{CH}_{3} \mathrm{CN}$ at $10^{\circ} \mathrm{C}$ was added freshly prepared $\mathrm{MgBr}_{2} \cdot \mathrm{Et}_{2} \mathrm{O}$. The reaction was stirred for $6 \mathrm{~h}$, quenched with satd. brine, extracted with $\mathrm{CHCl}_{3} / \mathrm{iPrOH}(2: 1)$, and dried with $\mathrm{Na}_{2} \mathrm{SO}_{4}$. The filtrate was concentrated affording $15 \mathrm{mg}(0.019 \mathrm{mmol}, 83$ $\%)$ of 21 as a white solid which was characterized without further purification. ${ }^{1} \mathrm{H}$ NMR (400 MHz, $\left.\left(\mathrm{CD}_{3}\right)_{2} \mathrm{CO}\right) \delta 7.75(\mathrm{~b}, 2 \mathrm{H})$, $7.60(\mathrm{~s}, 2 \mathrm{H}), 5.99(\mathrm{~d}, 2 \mathrm{H}, J=6.0 \mathrm{~Hz}), 5.09(\mathrm{~d}$, $2 \mathrm{H}, J=11.2 \mathrm{~Hz}), 4.37(\mathrm{dd}, 2 \mathrm{H}, J=11.2 \mathrm{~Hz}, J=$ $6.0 \mathrm{~Hz}), 3.89(\mathrm{~d}, 2 \mathrm{H}, J=18.4 \mathrm{~Hz}), 3.33(\mathrm{~m}$, 4H), $3.29(\mathrm{~d}, 2 \mathrm{H}, J=18.4 \mathrm{~Hz}), 1.60(\mathrm{~m}, 4 \mathrm{H}) ;{ }^{13} \mathrm{C} \mathrm{NMR}\left(75.0 \mathrm{MHz}, \mathrm{CDCl}_{3}\right) \delta 183.1,159.1$, 154.2, 148.8, 121.9, 90.9, 74.3, 56.7, 38.8, 38.0, 26.8; IR (thin film) vmax 3361, 2924, 2854, $1705,1658,1603,1541,1456,1377,1261,1095,1024 \mathrm{~cm}^{-1}$; ESIMS calculated for $\mathrm{C}_{22} \mathrm{H}_{22} \mathrm{Br}_{4} \mathrm{~N}_{4} \mathrm{O}_{8}\left[\mathrm{M}^{+}\right]$: 790.04, found: 790.8; $[\alpha]_{\mathrm{D}}{ }^{22}=-3.03\left(\mathrm{c}=0.006, \mathrm{CHCl}_{3}\right)$. 


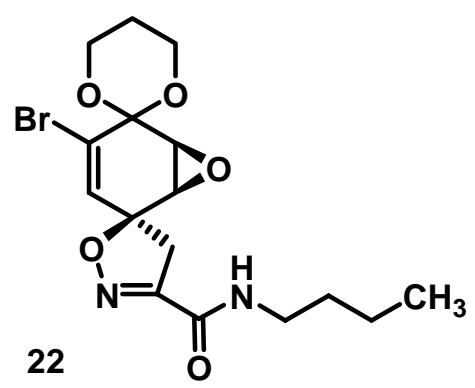

Monoamide ketal (22): Prepared from 13 in $72 \%$ yield as a yellow oil according to the procedure reported for monoamide 14. ${ }^{1} \mathrm{H}$ NMR $\left(300 \mathrm{MHz},\left(\mathrm{CD}_{3}\right)_{2} \mathrm{CO}\right) \delta 7.61(\mathrm{~b}$, $1 \mathrm{H}), 6.19(\mathrm{~d}, 1 \mathrm{H}, J=2.1 \mathrm{~Hz}), 4.42(\mathrm{~d}, 1 \mathrm{H}, J=4.2 \mathrm{~Hz}), 4.41-$ $4.32(\mathrm{~m}, 2 \mathrm{H}), 4.05-3.96(\mathrm{~m}, 2 \mathrm{H}), 3.70(\mathrm{dd}, 1 \mathrm{H}, J=1.5, J=$ $4.2 \mathrm{~Hz}) 3.41(\mathrm{~d}, 1 \mathrm{H}, J=18.3 \mathrm{~Hz}), 3.32(\mathrm{q}, 2 \mathrm{H}, J=7.2 \mathrm{~Hz})$, $3.25(\mathrm{~d}, 1 \mathrm{H}, J=18.3 \mathrm{~Hz}), 2.15-2.06(\mathrm{~m}, 1 \mathrm{H}), 1.65-1.49(\mathrm{~m}$, $3 \mathrm{H}), 1.41-1.22(\mathrm{~m}, 2 \mathrm{H}), 0.93(\mathrm{t}, 3 \mathrm{H}, \mathrm{J}=7.2 \mathrm{~Hz}) ;{ }^{13} \mathrm{C} \mathrm{NMR}$ (75.0 MHz, $\left.\left(\mathrm{CD}_{3}\right)_{2} \mathrm{CO}\right) \delta 158.9,154.2,130.4,125.7,92.6$, $86.5,61.2,60.8,54.2,48.9,42.9,38.9,31.6,24.6,19.9,13.3$;

IR (thin film) vmax 3320, 2959, 2931, 2873, 1665, 1599, 1537, 1467, 1433, 1383, 1260, $1142,1104,1044,1018 \mathrm{~cm}^{-1}$; CIHRMS [M] calculated for $\mathrm{C}_{16} \mathrm{H}_{21} \mathrm{BrN}_{2} \mathrm{O}_{5}: 400.0634$, found: $400.0650 ;[\alpha]_{\mathrm{D}}^{22}=+59.0\left(\mathrm{c}=0.010, \mathrm{CHCl}_{3}\right)$.

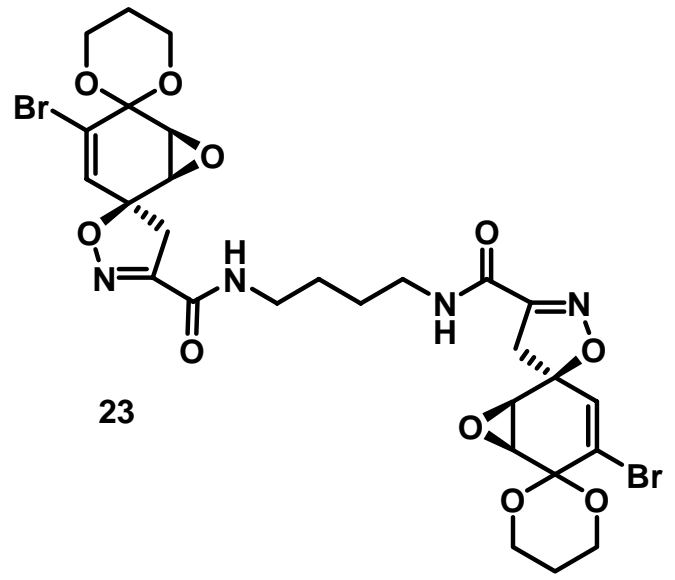

Bis-amide diketal (23): Prepared from 13 in $70 \%$ yield as a white solid according to the procedure reported for compound 17. M.p. 166$167{ }^{\circ} \mathrm{C} ;{ }^{1} \mathrm{H}$ NMR $\left(400 \mathrm{MHz},\left(\mathrm{CD}_{3}\right)_{2} \mathrm{CO}\right) \delta 7.63$ (br, 2H), $6.16(\mathrm{~d}, 2 \mathrm{H}, J=2.0 \mathrm{~Hz}), 4.38(\mathrm{~d}, 2 \mathrm{H}, J$ $=4.4 \mathrm{~Hz}), 4.37-4.30(\mathrm{~m}, 4 \mathrm{H}), 4.02-3.93(\mathrm{~m}, 4 \mathrm{H})$, $3.67(\mathrm{dd}, 2 \mathrm{H}, J=2.4,4.0 \mathrm{~Hz}), 3.38(\mathrm{~d}, 2 \mathrm{H}, J=$ $18.0 \mathrm{~Hz}), 3.31(\mathrm{q}, 4 \mathrm{H}, J=6.4 \mathrm{~Hz}), 3.22(\mathrm{~d}, 2 \mathrm{H}, J$ $=18.0 \mathrm{~Hz}), 2.11-2.03(\mathrm{~m}, 2 \mathrm{H}), 1.59-1.57(\mathrm{~m}$, $6 \mathrm{H}) ;{ }^{13} \mathrm{C}$ NMR $\left(75.0 \mathrm{MHz},\left(\mathrm{CD}_{3}\right)_{2} \mathrm{CO}\right) \delta 159.0$, 154.1, 130.4, 125.7, 92.5, 86.5, 61.3, 60.8, 54.2, $48.9,42.8,38.8,26.7,24.6$; IR (thin film) vmax 3377, 2923, 2360, 1656, 1453, 1367, 1143, 1041, $1005 \mathrm{~cm}^{-1}$; ESI-HRMS calculated for $\mathrm{C}_{28} \mathrm{H}_{32} \mathrm{Br}_{2} \mathrm{~N}_{4} \mathrm{O}_{10} \mathrm{Na}[\mathrm{M}+\mathrm{Na}]^{+}: 765.0383$, found: $765.0396[\alpha]_{\mathrm{D}}{ }^{22}=+50.0(\mathrm{c}=0.008$, $\left.\mathrm{CHCl}_{3}\right)$. 


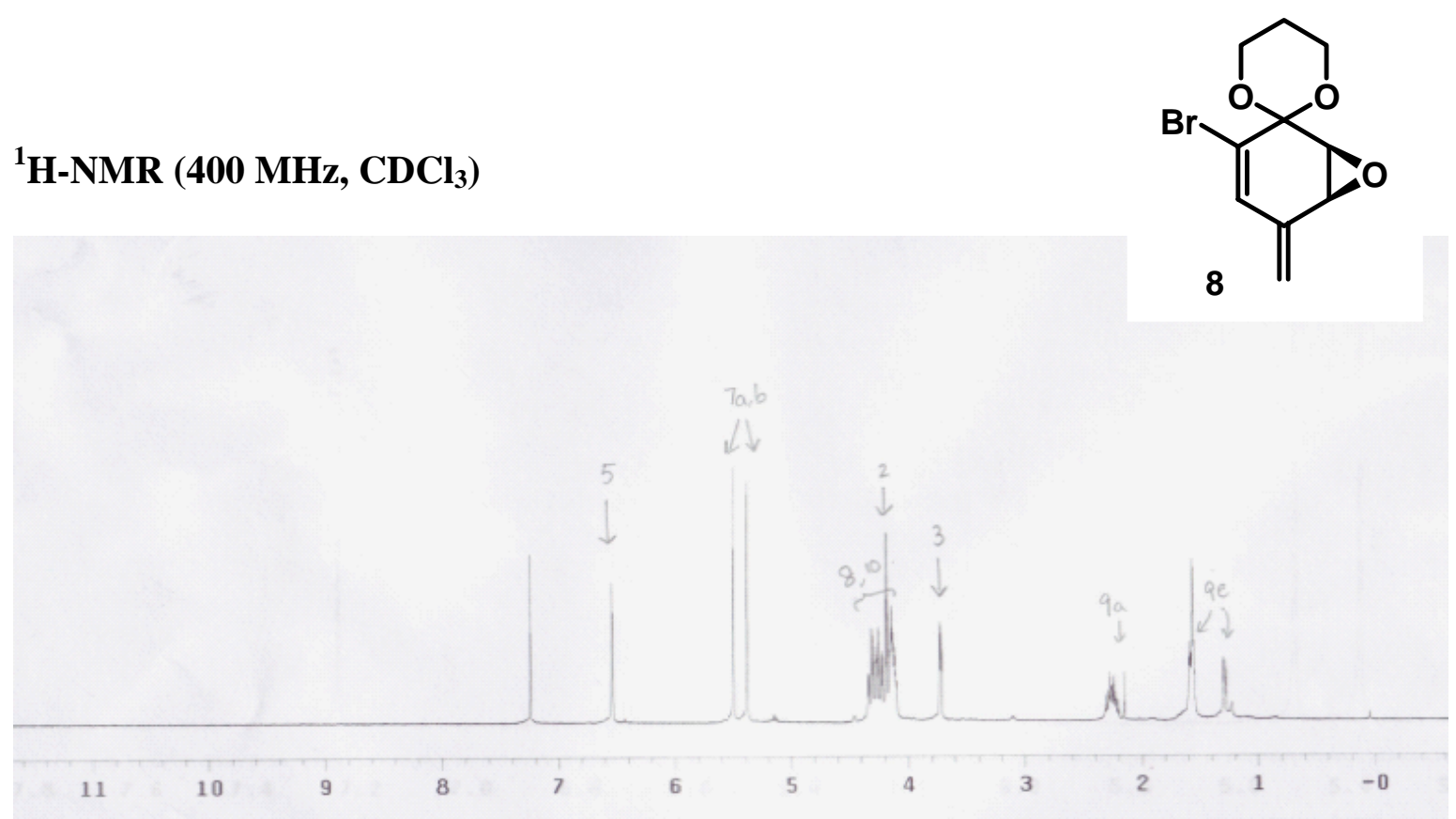

${ }^{13}$ C-NMR (100 MHz, $\mathrm{CDCl}_{3}$ )

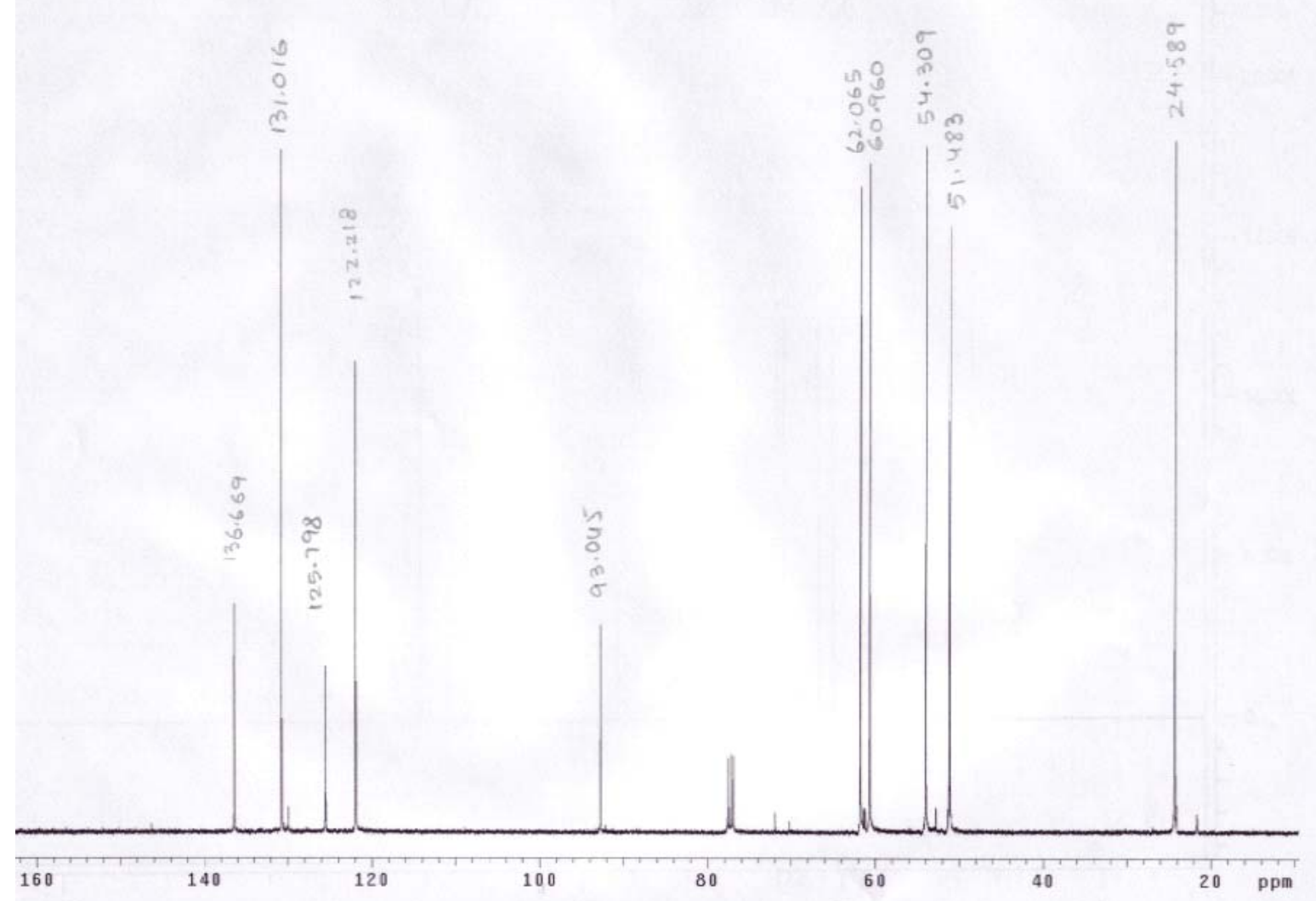


${ }^{1} \mathrm{H}-\mathrm{NMR}$ (300 MHz, $\mathrm{CDCl}_{3}$ )

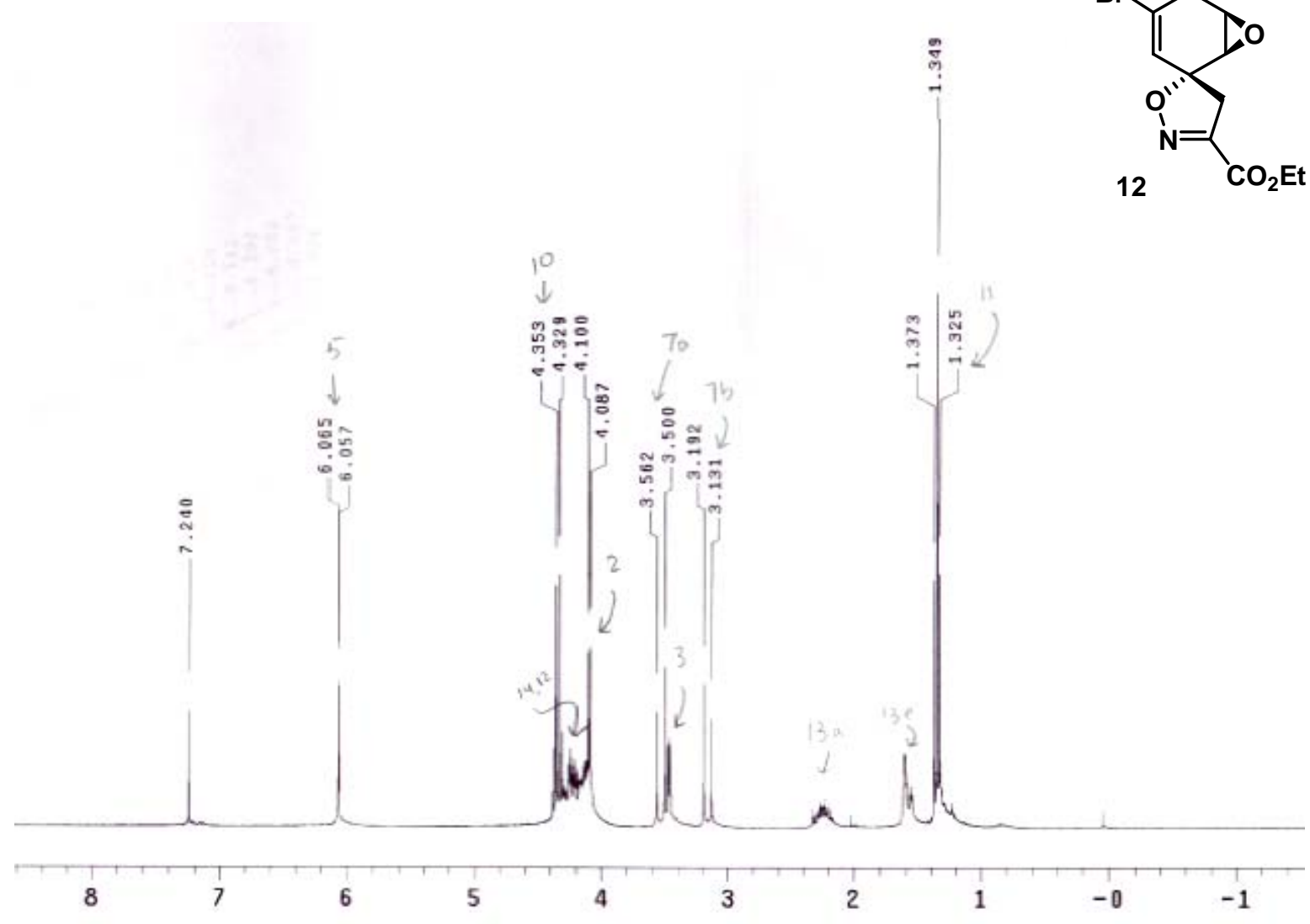

${ }^{13} \mathrm{C}-\mathrm{NMR}$ (75.0 MHz, $\mathrm{CDCl}_{3}$ )

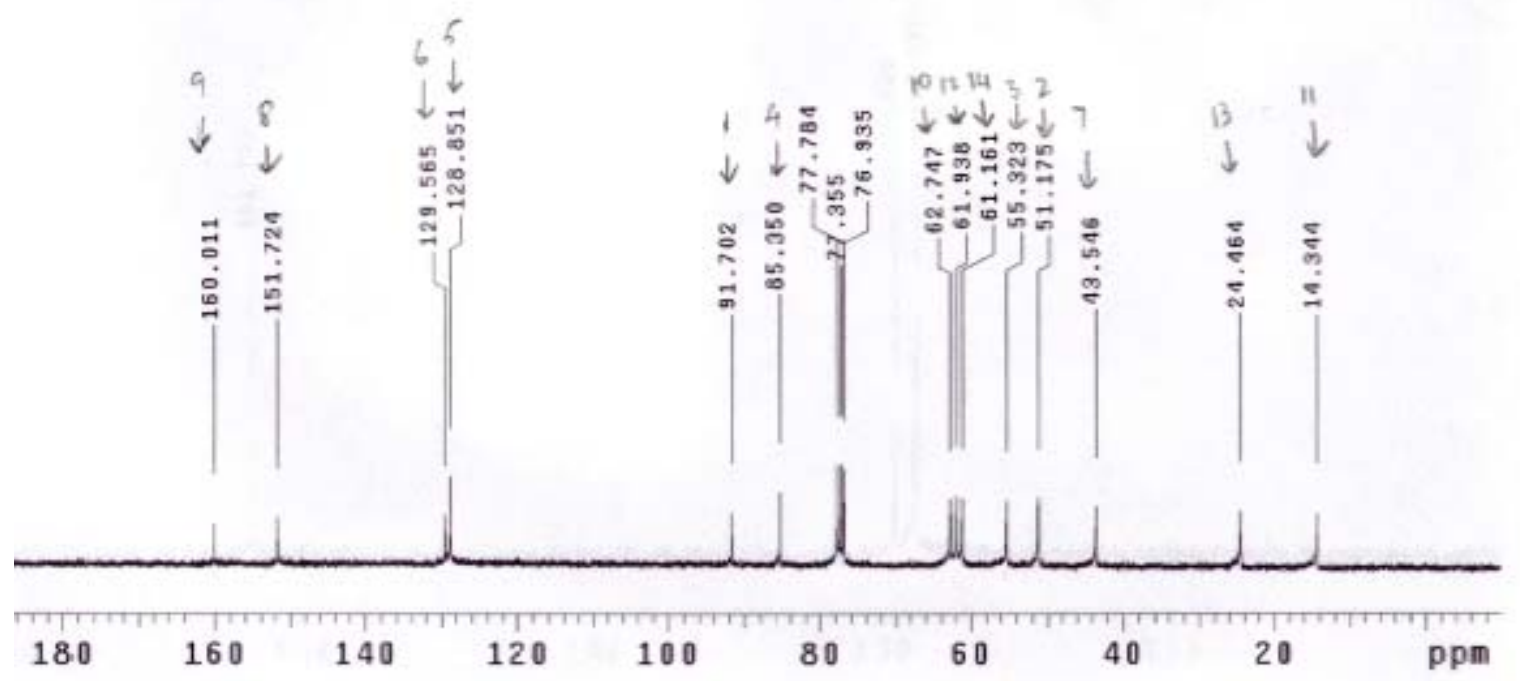




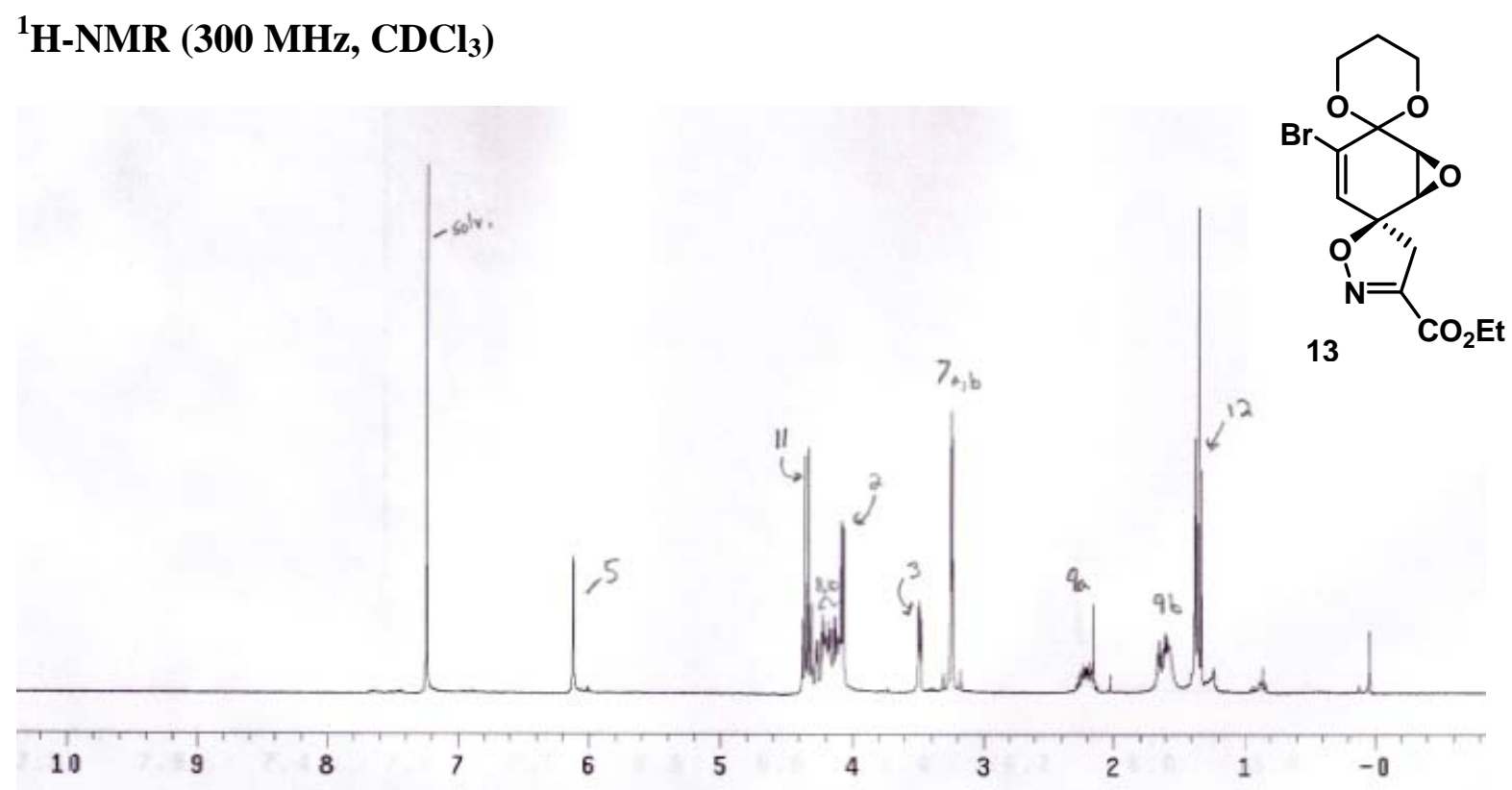

${ }^{13} \mathrm{C}-\mathrm{NMR}$ (75.0 MHz, $\mathrm{CDCl}_{3}$ )

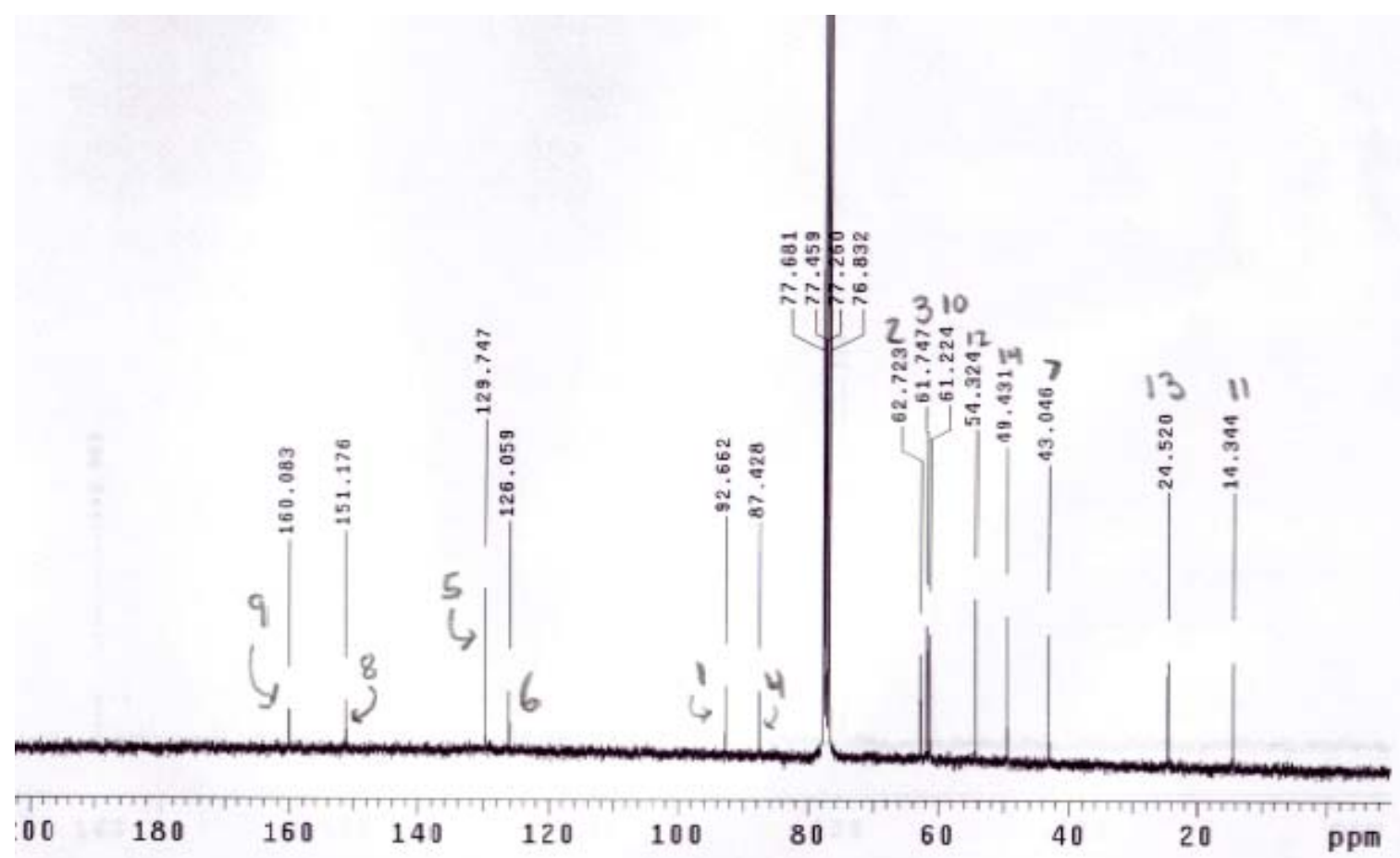




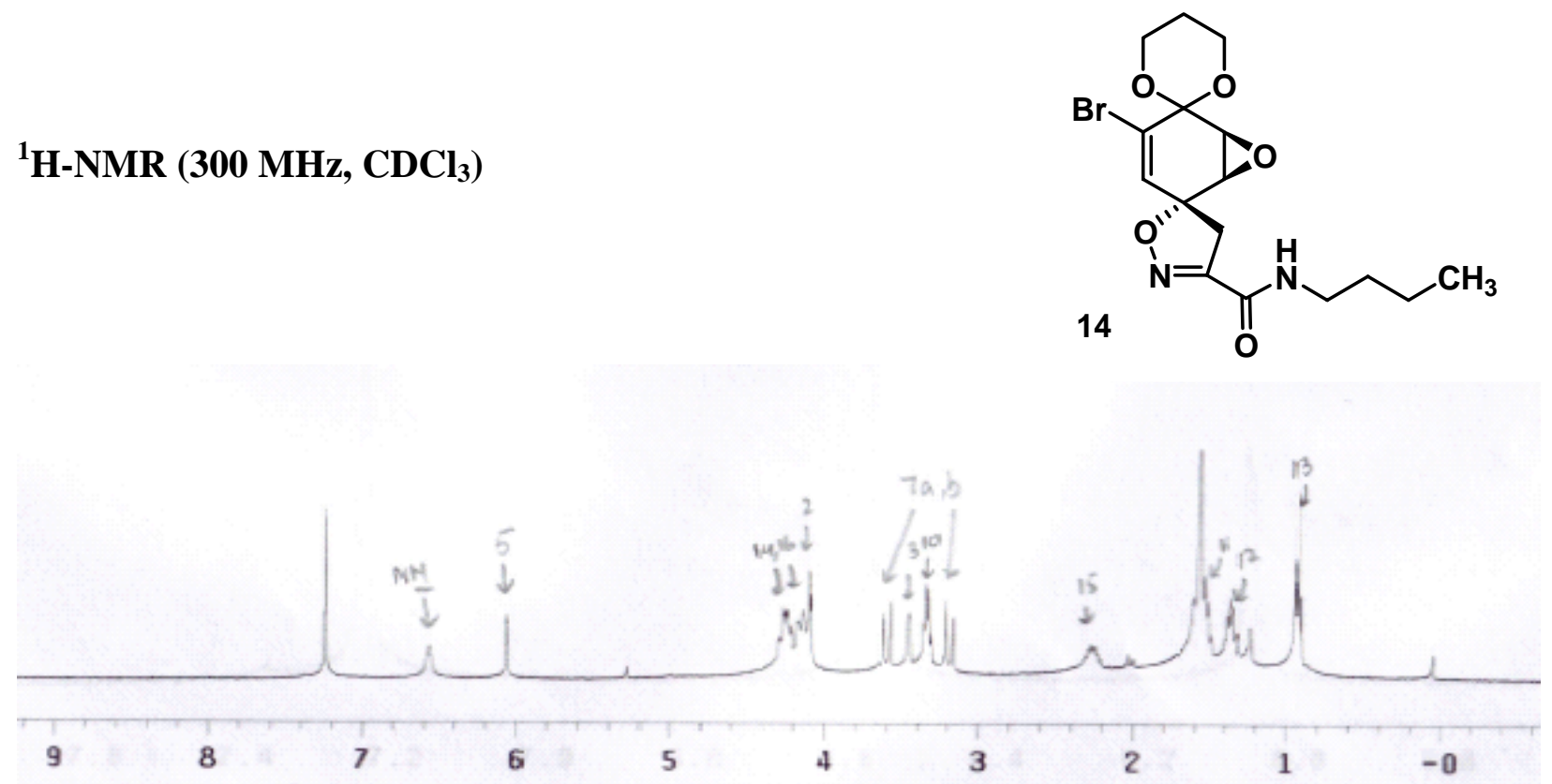

${ }^{13} \mathrm{C}-\mathrm{NMR}$ (75.0 MHz, $\left.\left(\mathrm{CD}_{3}\right)_{2} \mathrm{CO}\right)$

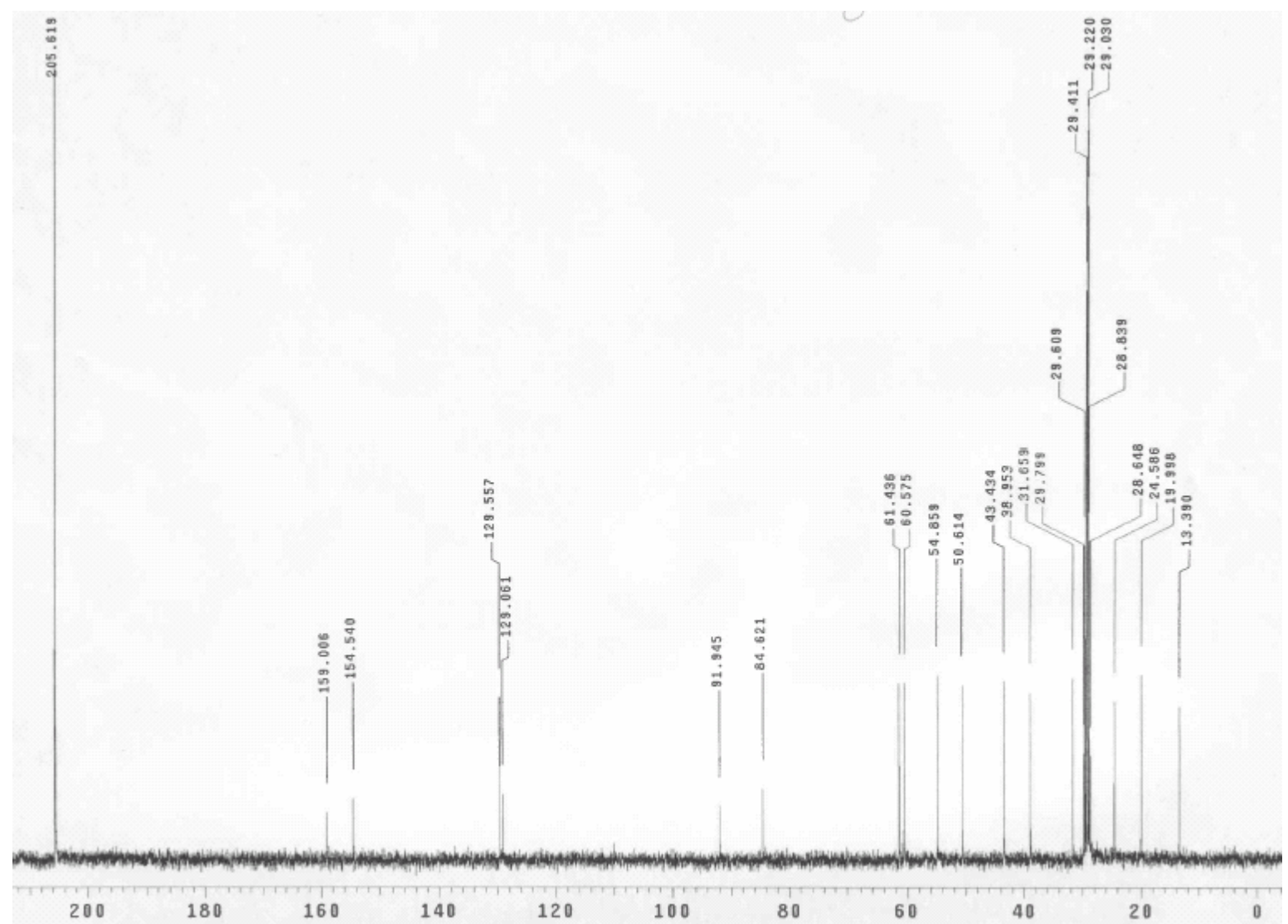



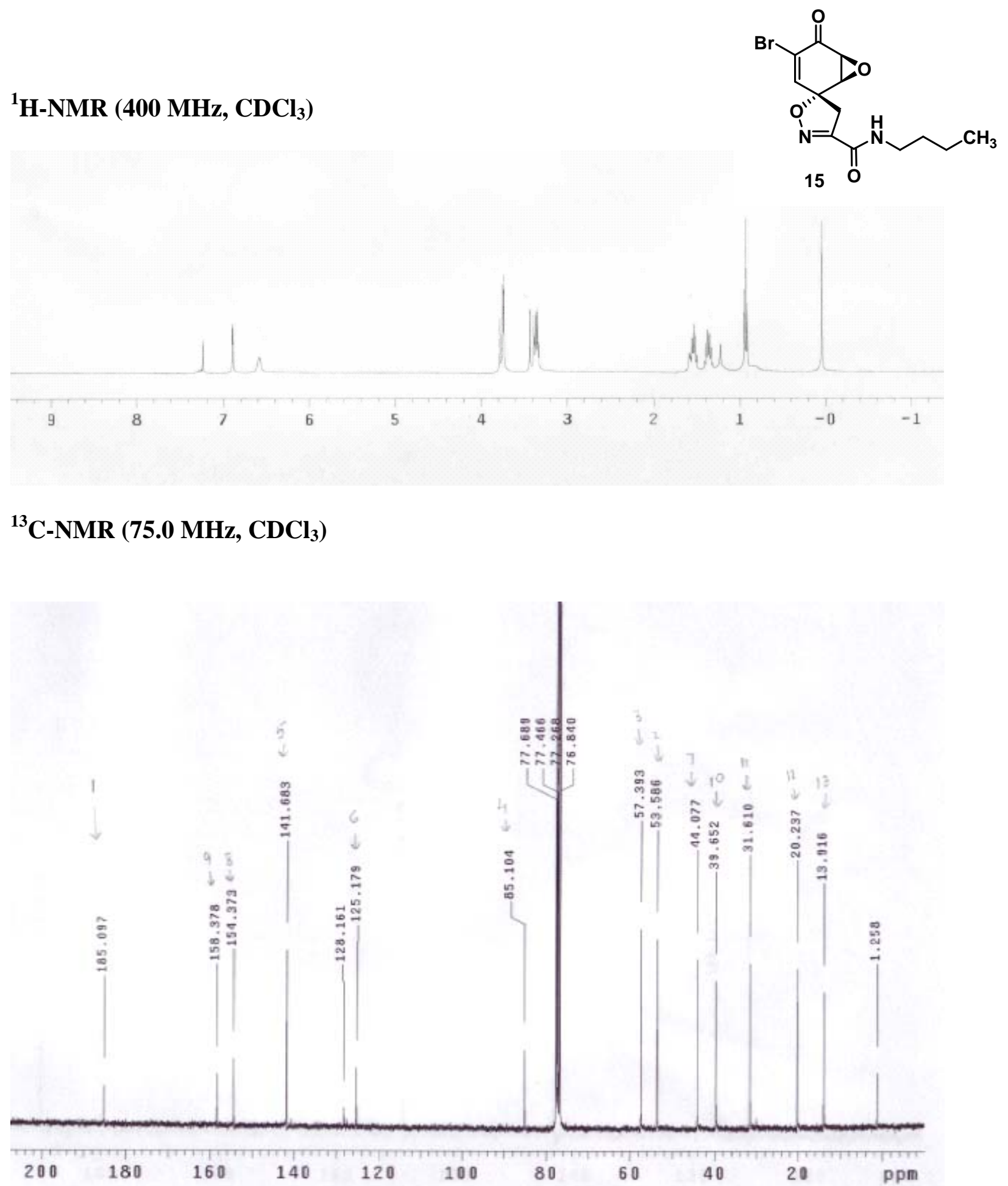


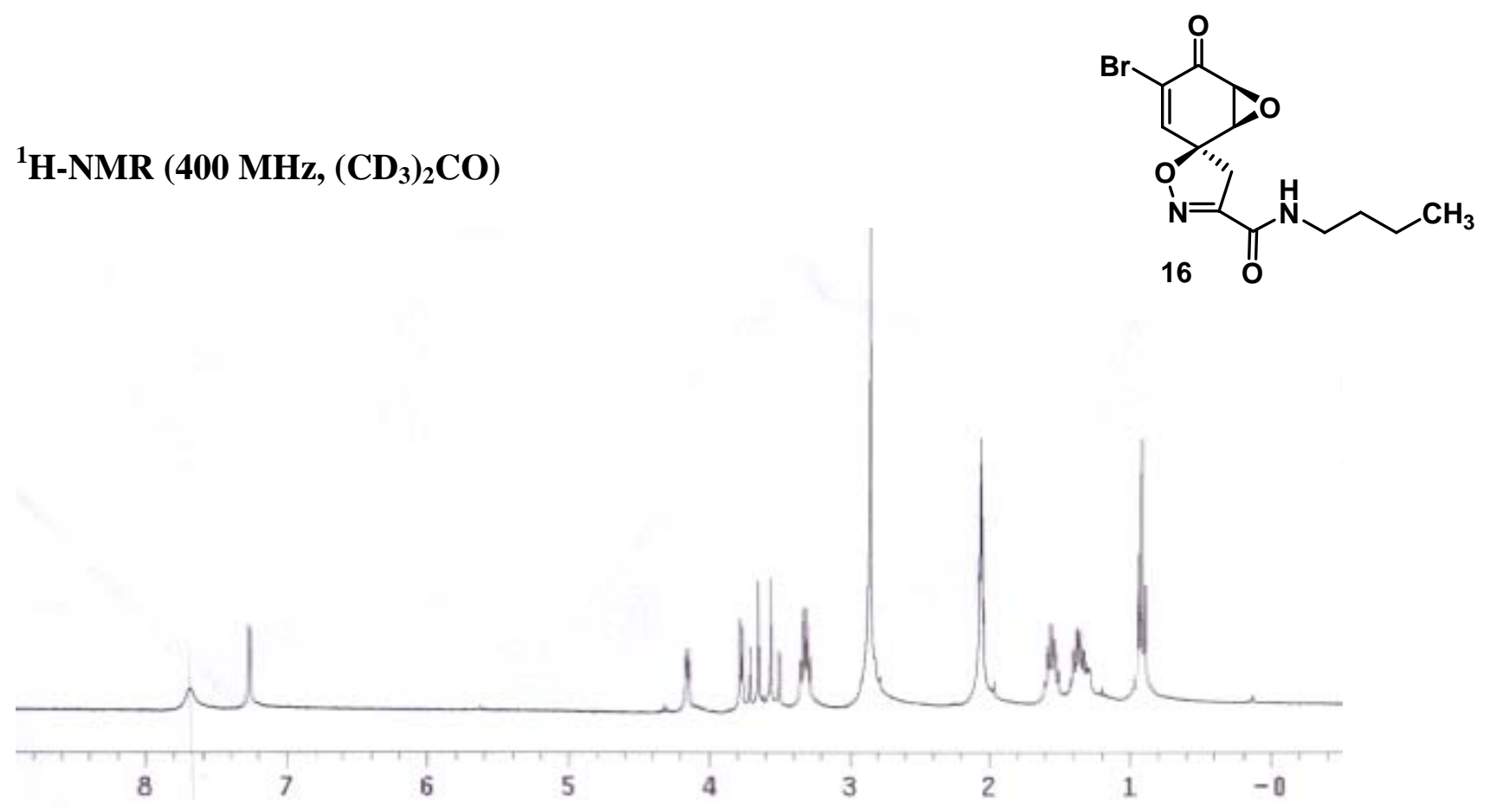

${ }^{13} \mathrm{C}-\mathrm{NMR}\left(75.0 \mathrm{MHz},\left(\mathrm{CD}_{3}\right)_{2} \mathrm{CO}\right)$

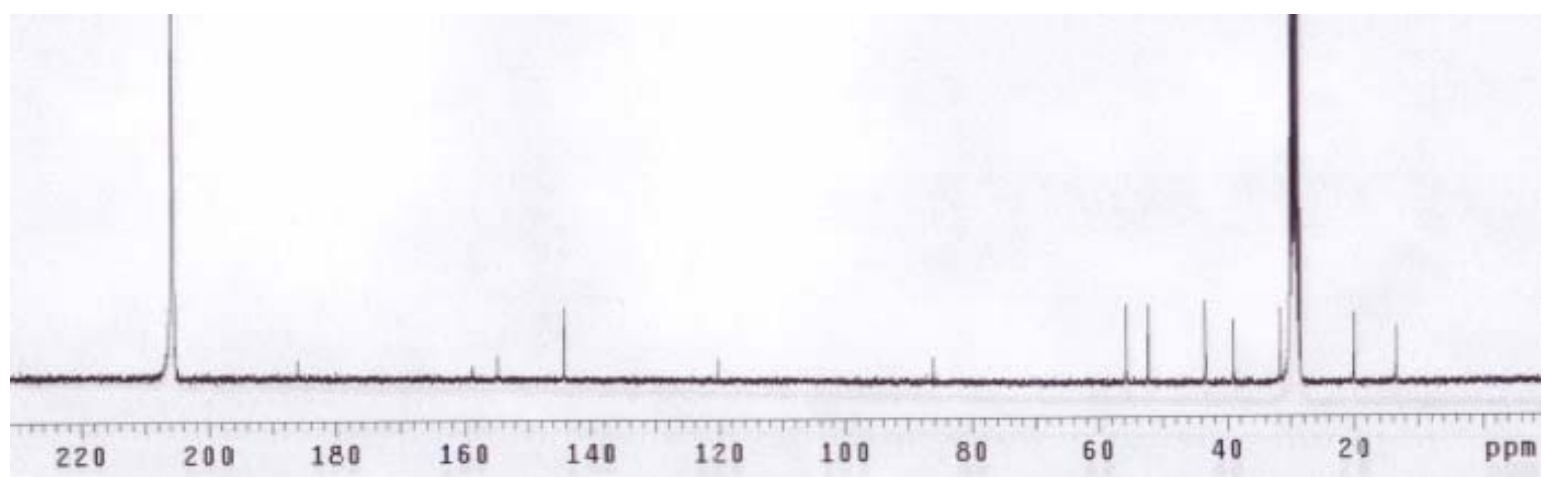




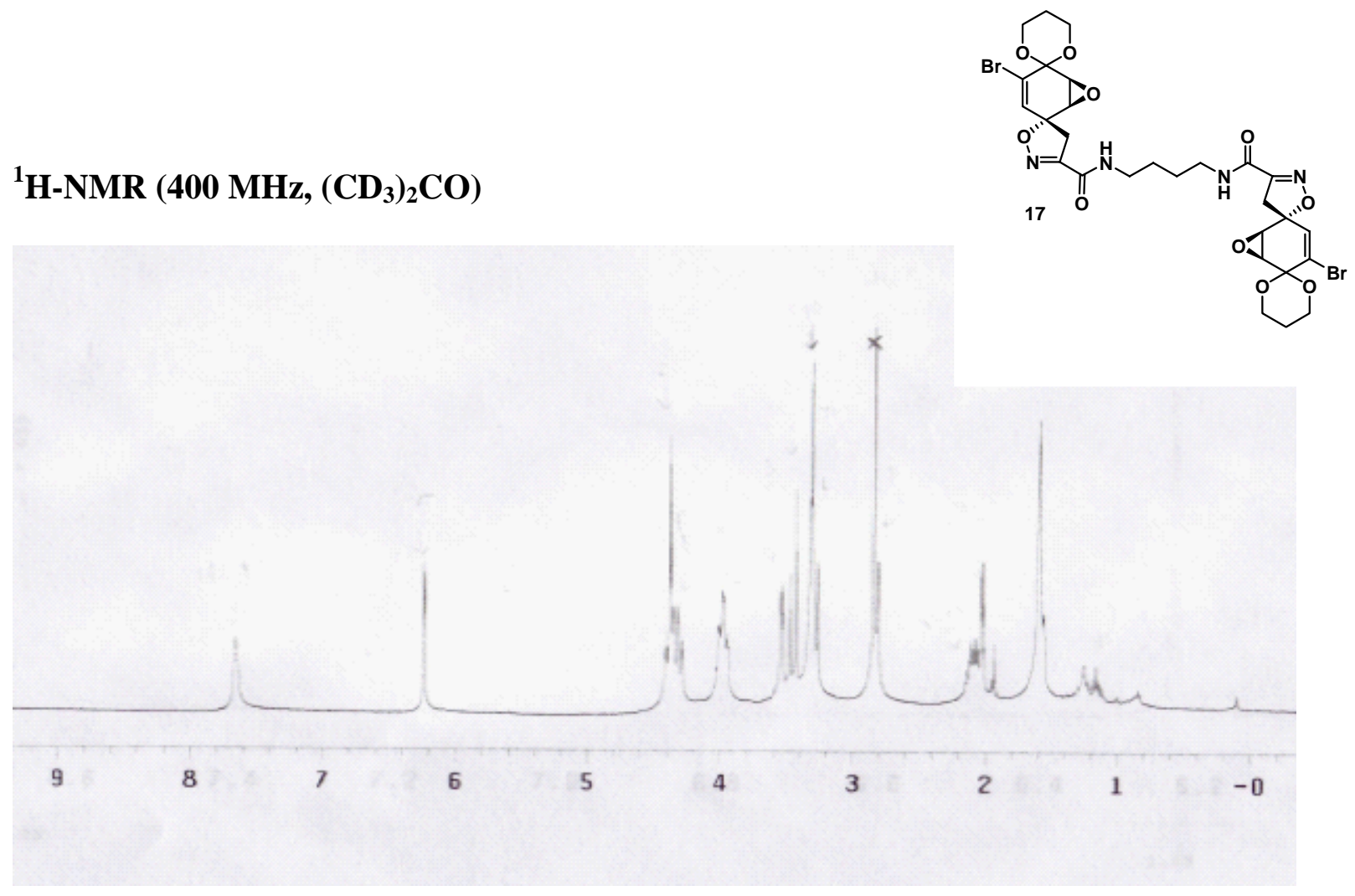

${ }^{13} \mathrm{C}-\mathrm{NMR}$ (75.0 MHz, $\left.\left(\mathrm{CD}_{3}\right)_{2} \mathrm{CO}\right)$

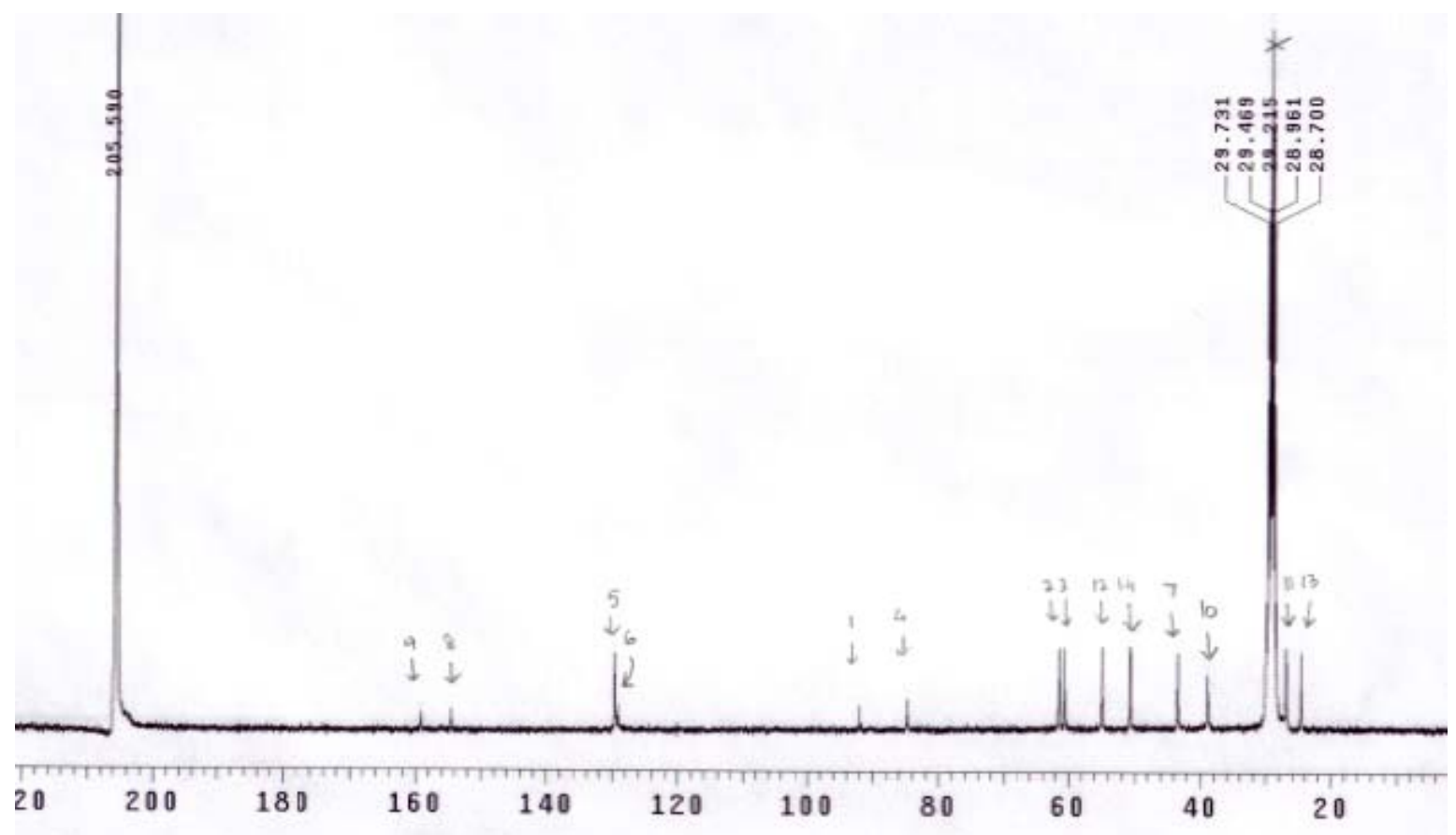




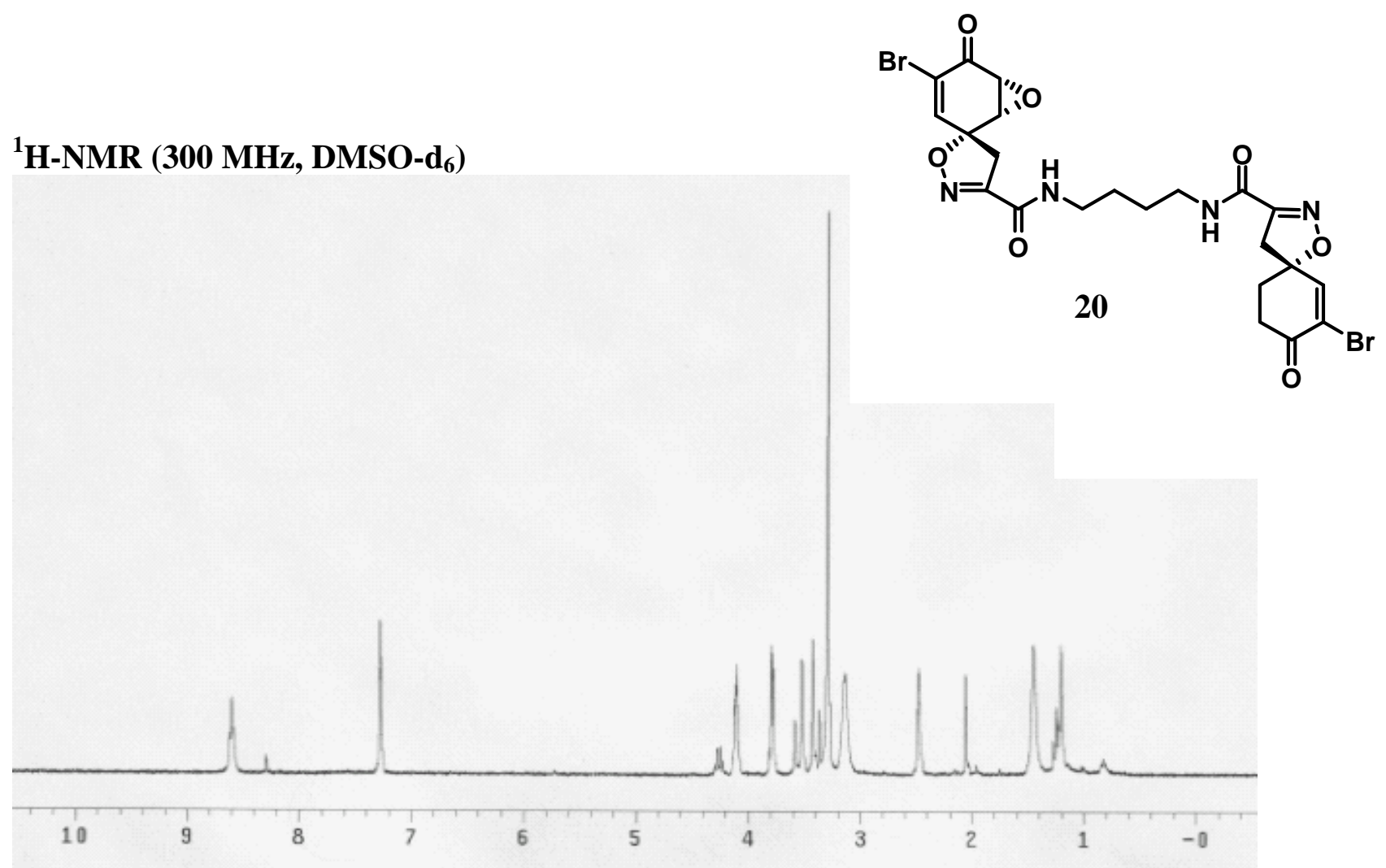

${ }^{13}$ C-NMR (75.0 MHz, DMSO-d ${ }_{6}$ )

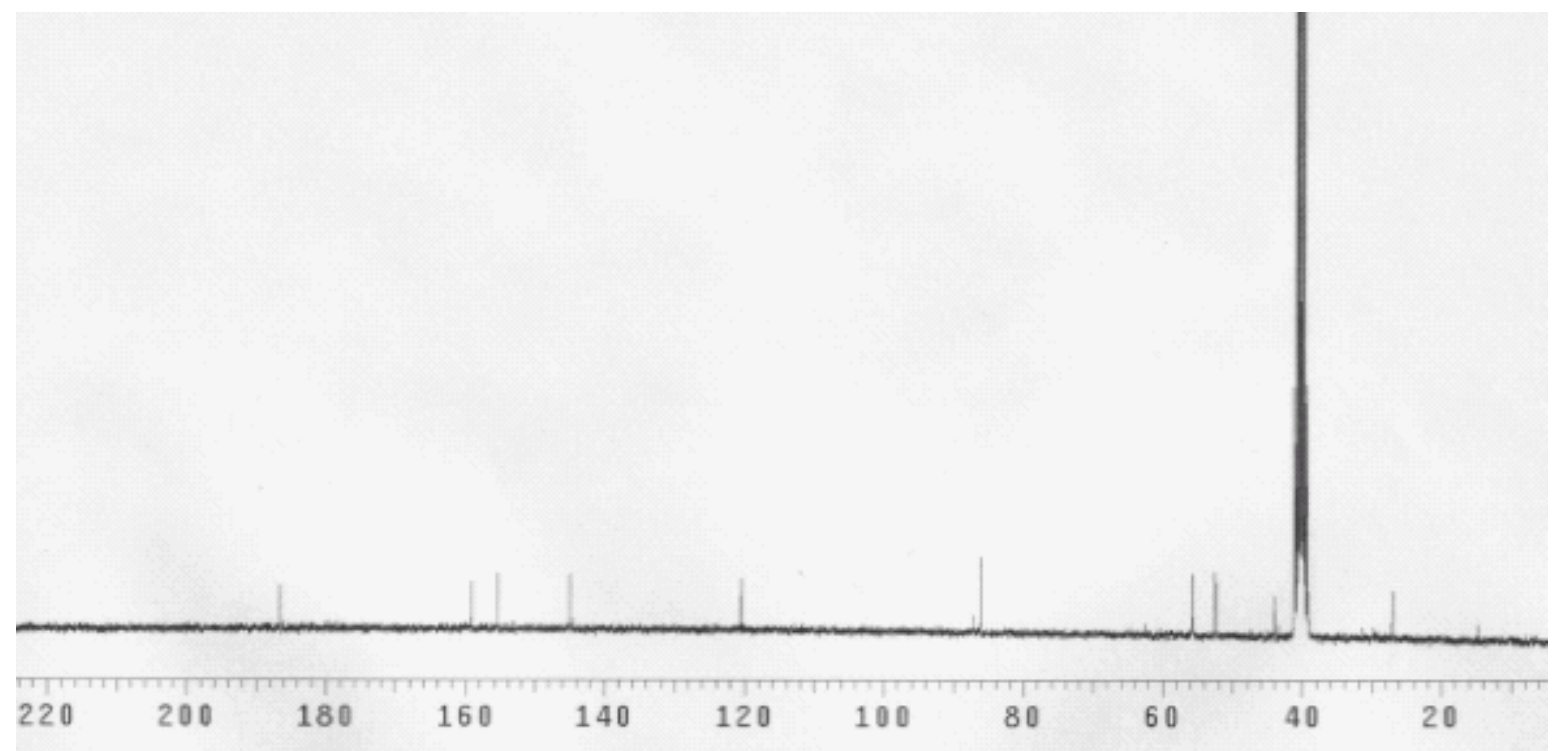




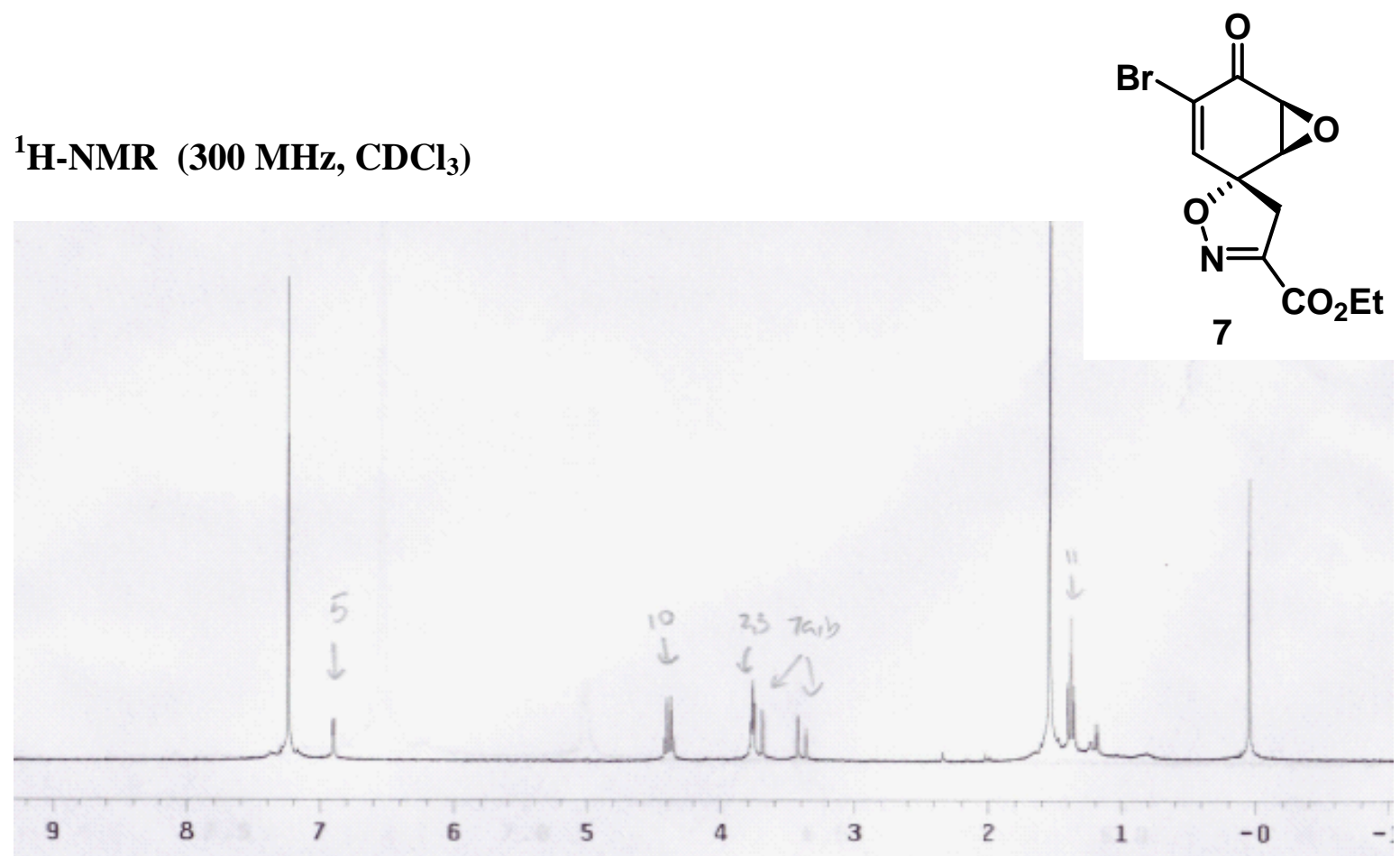

${ }^{13} \mathrm{C}-\mathrm{NMR}$ (75.0 MHz, $\mathrm{CDCl}_{3}$ )

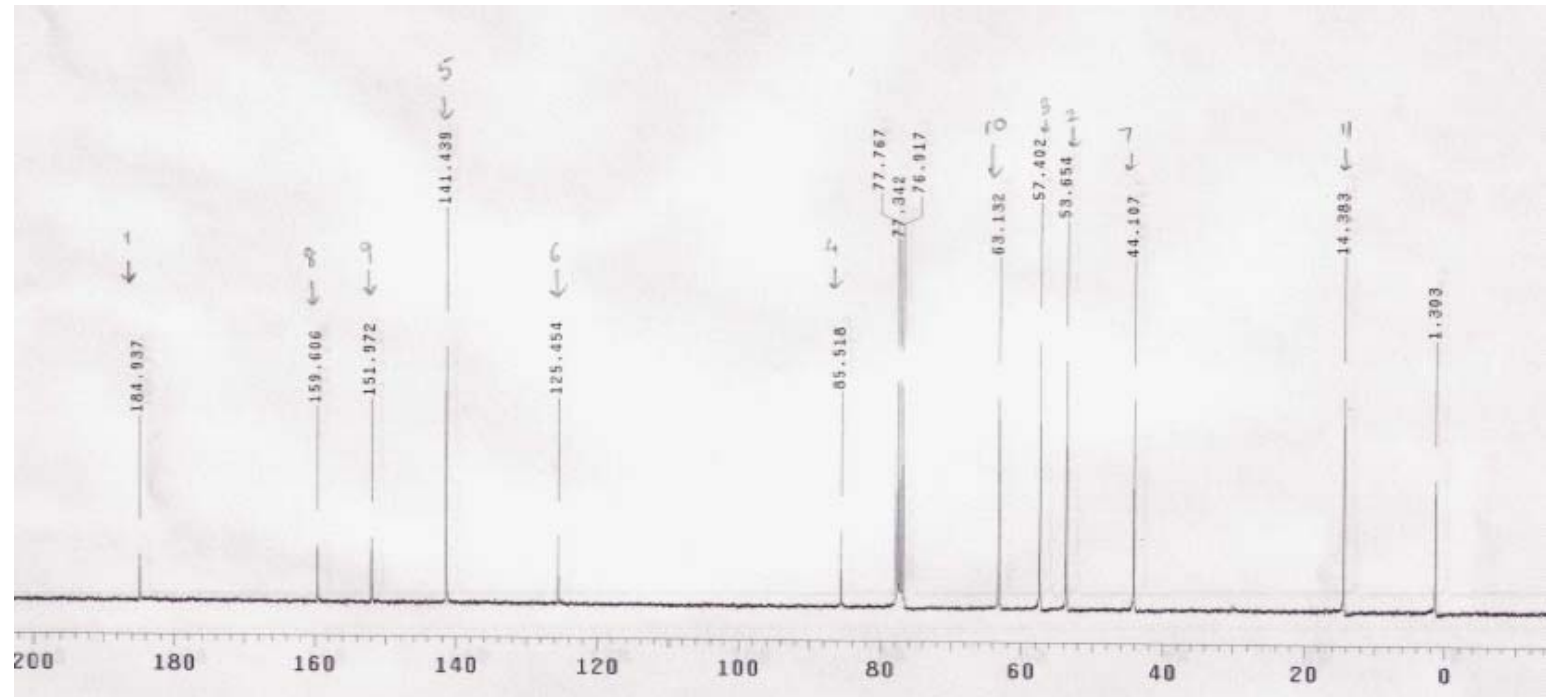


${ }^{1}$ H-NMR (400 MHz, $\mathrm{CDCl}_{3}$ )

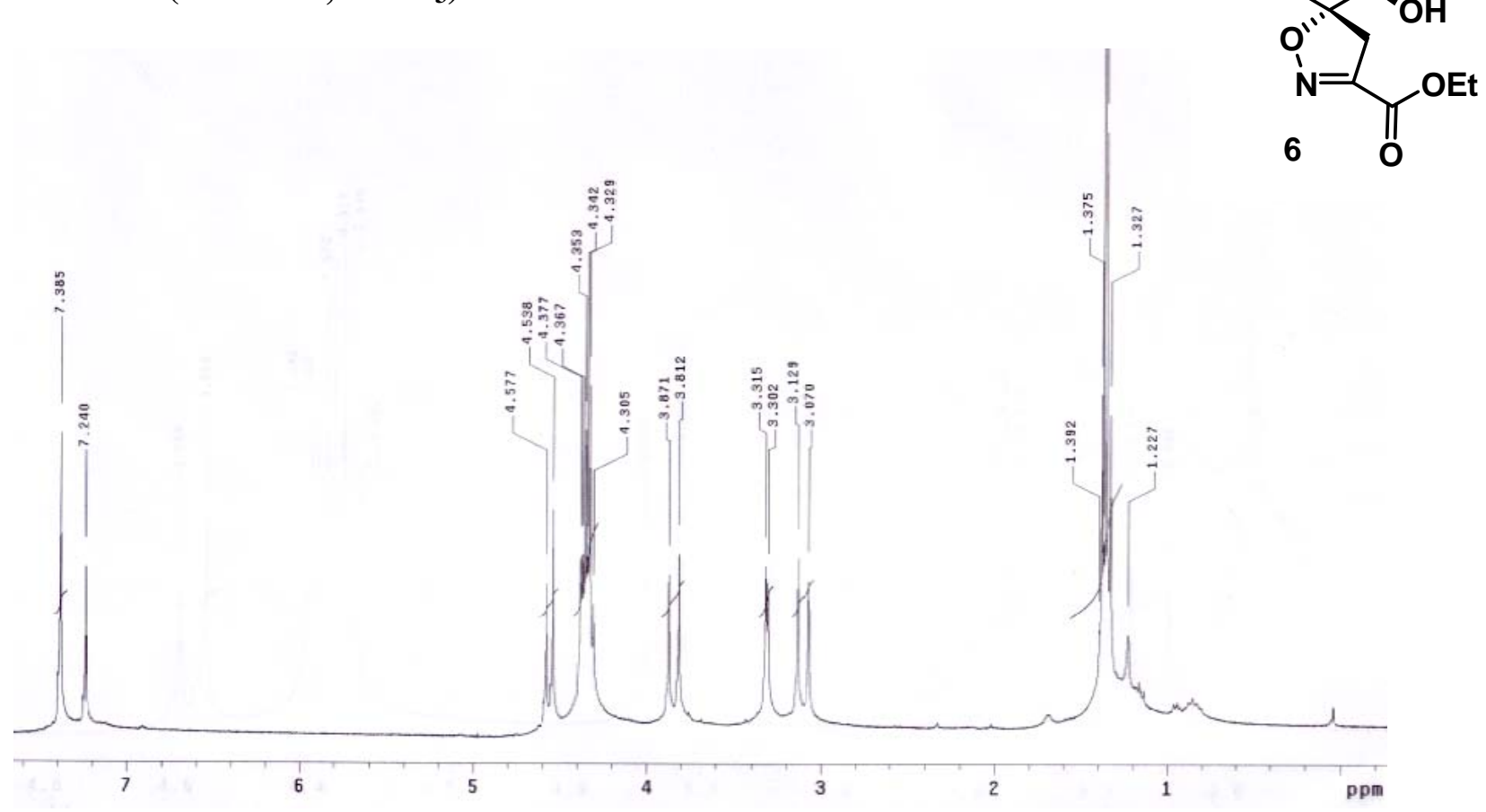

${ }^{13} \mathrm{C}-\mathrm{NMR}$ (75.0 MHz, $\mathrm{CDCl}_{3}$ )

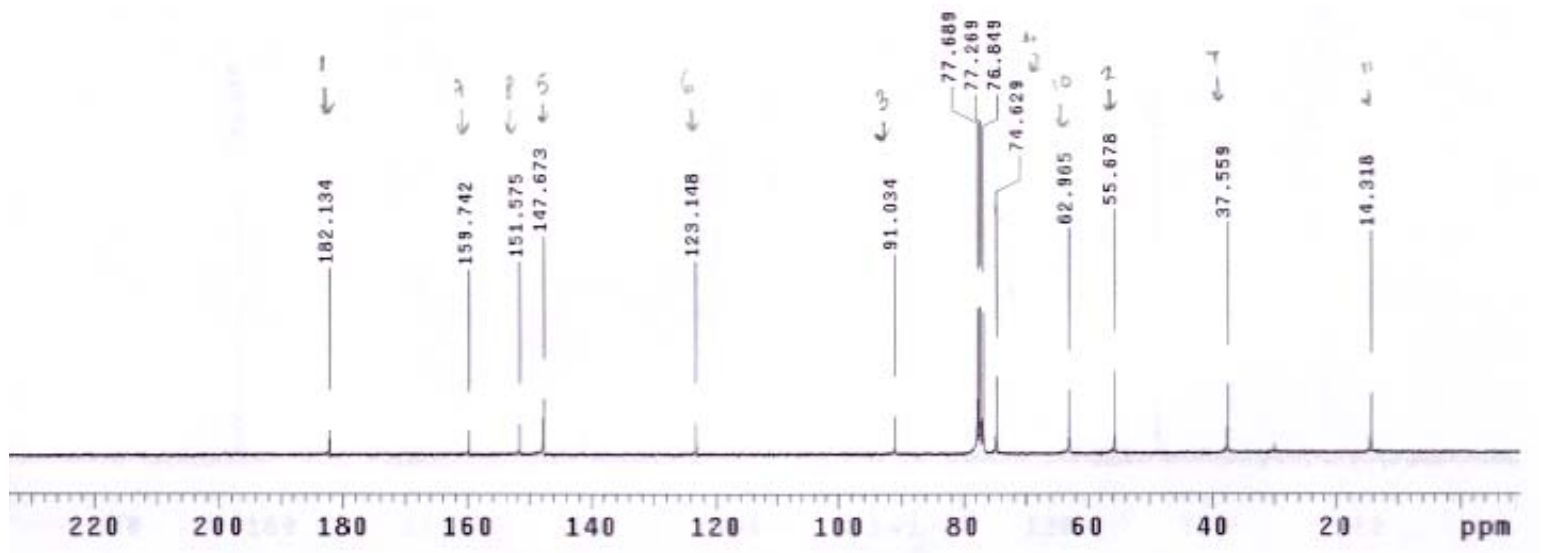




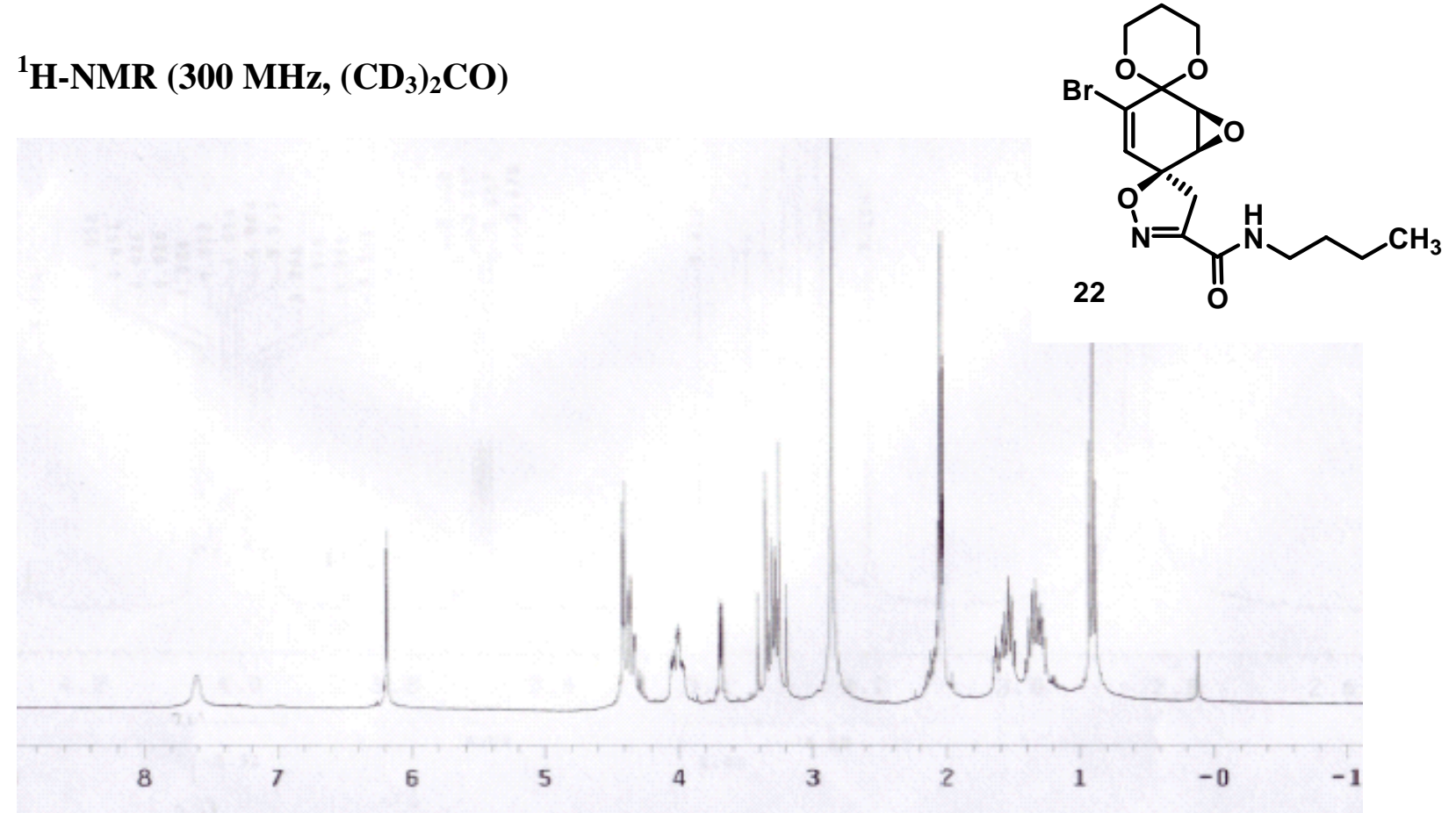

${ }^{13} \mathrm{C}-\mathrm{NMR}$ (75.0 MHz, $\left.\left(\mathrm{CD}_{3}\right)_{2} \mathrm{CO}\right)$

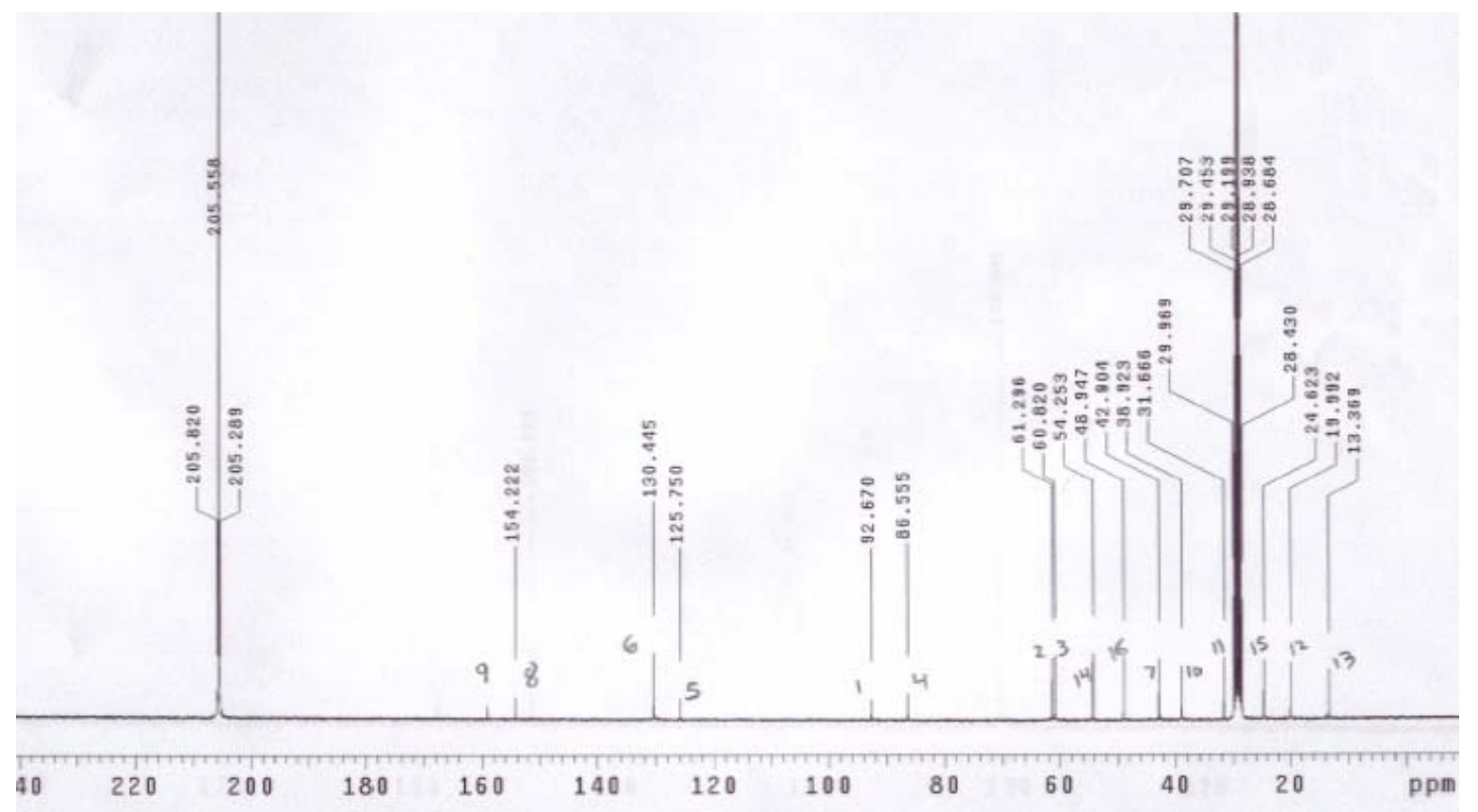




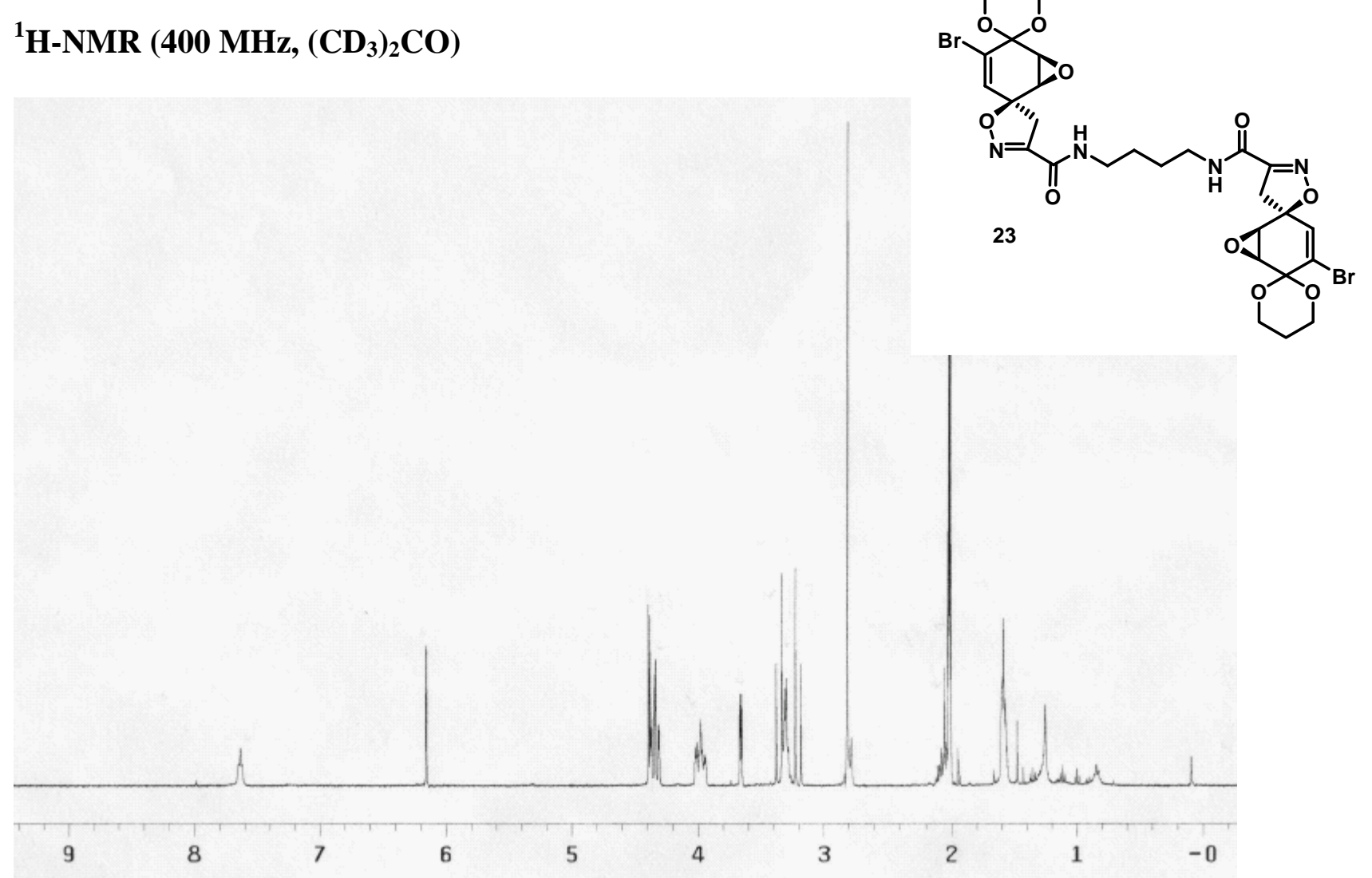

${ }^{13} \mathrm{C}-\mathrm{NMR}$ (75.0 MHz, $\left.\left(\mathrm{CD}_{3}\right)_{2} \mathrm{CO}\right)$

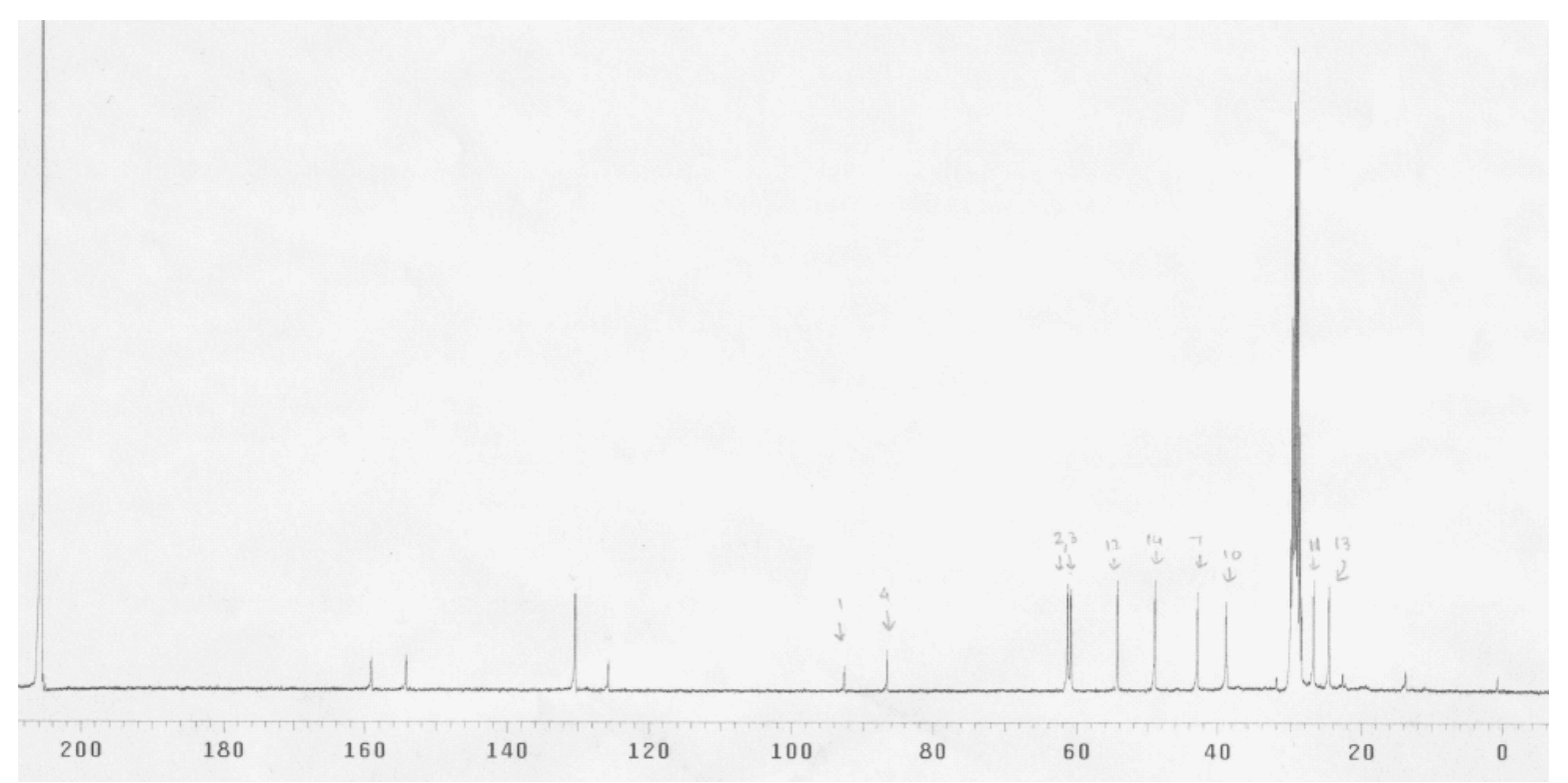

